\title{
Maximal closed ideals of the Colombeau Algebra of Generalized functions
}

\author{
A. Khelif ${ }^{1} \cdot$ D. Scarpalezos ${ }^{1}$
}

Received: 25 March 2019 / Accepted: 15 October 2020 / Published online: 28 October 2020

(c) The Author(s) 2020

\begin{abstract}
In this paper we investigate the structure of the set of maximal ideals of $\mathcal{G}(\Omega)$. The method of investigation passes through the use of the $m$-reduction and the ideas are analoguous to those in Gillman and Jerison (Rings of Continuous Functions, N.J. Van Nostrand, Princeton, 1960) for the investigation of maximal ideals of continuous functions on a Hausdorff space $K$.
\end{abstract}

Keywords Ideal $\cdot$ Net $\cdot$ Ultrafilter $\cdot$ Functions $\cdot$ Distributions $\cdot$ Algebra

Mathematics Subject Classification Primary 54C40 - 46F30; Secondary 46E10 . 46E25

\section{Introduction}

The algebra of Colombeau generalized functions has been used for non linear P.D.E. (see for example [4,6-8,22,24]) as well as for linear P.D.E. with irregular coefficients [21]. Much work has been done on this field from the point view of Analysis. However the Algebraic properties of this Algebra are still a largely open field of investigation.

In the case of continuous functions (with complex or real values over a compact set $K$ it is known that the set of points are in one-one correspondence with the set of maximal ideals. While in the more general case of the algebra of continuous functions on a Hausdorff regular space $A$, the set of maximal ideals is in a one to one correspondence with the Čech-compactification $(\beta(A))$ (see [16]).

It is known that generalized functions $\mathcal{G}(\Omega)$ have a double structure:

(a) They constitute a sheaf of algebras (see [4,6]) and

\section{Communicated by Adrian Constantin.}

\footnotetext{
$凶 \quad$ A. Khelif

khelif@math.univ-paris-diderot.fr

1 Institut de mathematiques de Jussieu Paris Rive Gauche, Paris, France
} 
(b) They are continuous mappings of $\widehat{\Omega}_{c}$ (the set of compactly supported generalized points) into the ring $\overline{\mathbb{K}}$ of generalized constants. (This result follows immediately from definitions.)

This leads to the notion of 'localisation' of ideals, the notion of 'support' of an ideal and to the notion of generalized trace of ideals (see [2]).

Trying to obtain a better knowledge of prime and maximal ideals of $\mathcal{G}(\Omega)$ we make an extensive use of the results concerning the algebraic and topological properties of the ring of generalized constants [3]. In order to use ideas analogous to those in [16], we first use the $m$-reduction procedure [27] which gives a canonical surjective mapping of $\mathcal{G}(\Omega)$ onto $\mathcal{G}_{m}(\Omega)$, but $\mathcal{G}_{m}(\Omega)$ is a topological algebra on the field of $m$-reduced generalized constants $\overline{\mathbb{K}}_{m}=\overline{\mathbb{K}} / m$ where $m$ is maximal ideal of $\overline{\mathbb{K}}$. We now consider a very large class of maximal ideals called regular maximal ideals. The set of regular maximal ideals is proved to contain all closed maximal ideals (closed for the natural Hausdorff topology of $\mathcal{G}(\Omega)$ ).

Our main result is the following: the set of regular maximal ideals is in one to one correspondence with $(m, d)$ where $m$ is a maximal ideal of $\overline{\mathbb{K}}$ and $d \in \gamma\left(\widehat{\Omega}_{m, c}\right)$ where $\gamma\left(\widehat{\Omega}_{m, c}\right)$ is a special compactification of $\widehat{\Omega}_{m, c}$ called the $g$-compactification of $\widehat{\Omega}_{m, c}$.

In Sect. 1. in order to make the paper almost self contained we give the main definitions and results that we will use.

In Sect. 2. we give some results and examples and counterexamples concerning the properties of ideals of $\mathcal{G}(\Omega)$.

In Sect. 3. with the help of the concept of $m$-reduction we investigate the set of maximal ideals of the algebra $\mathcal{G}_{m}(\Omega)$ and show how to transfer these results by pull back on $\mathcal{G}(\Omega)$.

In the appendix we present the technique of compactification with the help of special families of closed sets called compactifying families.

\section{Definitions and main results}

In order to make this paper almost self contained and accessible to non specialists we remind the main definition and the main algebraic results. In order to enlighten the functoriality of Colombeau constructions we will follow the presentation given in [26].

\subsection{Colombeau extension}

Definition 1.1 Let $\left(E,\left(\mu_{n}\right)_{n}\right)$ be a Hausdorff topological vector space whose uniform structure is given by an increasing sequence $\left(\mu_{n}\right)_{n}$ of seminorms.

A net $\left(f_{\varepsilon}\right)_{\varepsilon} \in E^{] 0,1]}$ is said to be moderate if

$$
\forall n \in \mathbb{N}, \exists a_{n} \in \mathbb{R} \text { s.t. } \mu_{n}\left(f_{\varepsilon}\right)=o\left(\varepsilon^{a_{n}}\right)
$$

The vector space of moderate nets will be denoted $\mathcal{E}_{M}[E]$. 
A net $\left(h_{\varepsilon}\right) \in E^{] 0,1]}$ will be said to be negligible if

$$
\forall n \in \mathbb{N}, \forall b \in \mathbb{R}, \mu_{n}\left(h_{\varepsilon}\right)=o\left(\varepsilon^{b}\right)
$$

The subspace of all negligible nets will be denoted $\mathcal{N}[E]$.

The factor space $\mathcal{G}[E]=\mathcal{E}_{M}[E] / \mathcal{N}[E]$ will be called the Colombeau extension of E.

The class of a moderate net $\left(f_{\varepsilon}\right)_{\varepsilon}$ will be denoted $\left[\left(f_{\varepsilon}\right)_{\varepsilon}\right]$. Notice that the above construction depends only on the uniform structure of $E$ and not of the precise sequence $\left(\mu_{n}\right)$ chosen to express it.

Definition 1.2 If $\left(f_{\varepsilon}\right)_{\varepsilon} \in \mathcal{E}_{M}[E]$ we define the valuations

$$
v_{n}\left(f_{\varepsilon}\right):=\sup \left\{a \in \mathbb{R} \text { s.t. } \mu_{n}\left(f_{\varepsilon}\right)=o\left(\varepsilon^{a}\right)\right\}
$$

Clearly $\left(f_{\varepsilon}\right)_{\varepsilon} \in \mathcal{N}[E]$ if and only if $\forall n, v_{n}\left(f_{\varepsilon}\right)=+\infty$. We also define a family of pseudodistances by:

$$
\delta_{n}\left(\left(f_{\varepsilon}\right),\left(g_{\varepsilon}\right)\right)=e^{-v_{n}\left(\left(f_{\varepsilon}-g_{\varepsilon}\right)_{\varepsilon}\right)}
$$

as $f_{\varepsilon}-g_{\varepsilon} \in \mathcal{N}[E]$ if and only if

$$
\forall n \delta_{n}\left(\left(f_{\varepsilon}\right),\left(g_{\varepsilon}\right)\right)=0
$$

Those notions pass to the factor space $\mathcal{G}[E]=\mathcal{E}_{M}[E]_{/ \mathcal{N}[E]}$ and define on it a complete Hausdorff ultrametric uniform structure. The topology they define on $\mathcal{G}[E]$ is called the sharp topology (see $[14,15,21,25,26]$ ). This notion has been extended to many analogous constructions (see $[9,10,12,13])$.

The above extension procedure is functorial in the sense that if $L: E \rightarrow F$ is a continuous linear mapping, then $L$ defines in an obvious way a mapping $L_{g}: \mathcal{G}[E] \rightarrow$ $\mathcal{G}[F]$ which is continuous for respective sharp topologies. This property holds also for a larger class of mappings the so called 'continuously tempered' mappings $([9,26])$. This class contains bilinear and quadratic mappings; thus if $E$ is a topological algebra on $\mathbb{K}$, then $\mathcal{G}[E]$ is a topological algebra on the Colombeau extension of $\mathbb{K}$ that we will denote $\overline{\mathbb{K}}$.

\subsection{Generalized constants}

Definition 1.3 The Colombeau extension of the field $\mathbb{K}(\mathbb{K}=\mathbb{R}$ or $\mathbb{C}$ ) endowed with its absolute value will be denoted by $\overline{\mathbb{K}}$, the ring of generalized constants.

The trivial embedding $\left.\left.\left(x \rightarrow\left[\left(x_{\varepsilon}\right)\right], x_{\varepsilon}=x, \forall \varepsilon \in\right] 0,1\right]\right)$ of $\mathbb{K}$ into $\overline{\mathbb{K}}$ is discrete (the same holds generally for all trivial embeddings $E \rightarrow \mathcal{G}[E]$ ).

The ring $\overline{\mathbb{K}}$ of generalized constants is not a field. Its main algebraic and topological properties have been studied in [3], and later in [5,8,28]. Let us cite the main results we will use in this paper: 
1) $\overline{\mathbb{C}}=\overline{\mathbb{R}}+i \overline{\mathbb{R}}$ and if $a$ is an ideal of $\overline{\mathbb{C}}$ then $a=(a \cap \overline{\mathbb{R}})+i(a \cap \overline{\mathbb{R}})$.

2) If $p$ is a prime ideal of $\overline{\mathbb{K}}$ then its closure $\bar{p}$ (closure for the sharp topology) is a maximal ideal of $\overline{\mathbb{K}}$.

3) If in $\overline{\mathbb{R}}$ we define $x \leq y$ by the fact that

there exist respective representatives $\left(x_{\varepsilon}\right)_{\varepsilon}$ and $\left(y_{\varepsilon}\right)_{\varepsilon}$ s.t. $\forall \varepsilon\left(x_{\varepsilon}\right) \leq\left(y_{\varepsilon}\right)$

then we define an order on $\overline{\mathbb{R}}$ (but not a total order).

4) Let us denote by $|x|$ the class of $\left(\left|x_{\varepsilon}\right|\right)_{\varepsilon}$. If $\mathcal{U}$ is an ideal of $\overline{\mathbb{K}},|y| \in \mathcal{U}$ and $|x| \leq|y|$, then $x \in \mathcal{U}$.

\subsection{Generalized functions}

Let us consider the space $E=C^{\infty}(\Omega)$ where $\Omega$ is an open subset of $\mathbb{R}^{d}$. Let us remind first how we can define the uniform structure of $E$ by an increasing sequence of seminorms.

Let $\left(\Omega_{n}\right)_{n}$ be an exhaustive sequence of relatively compact subsets of $\Omega$ (i.e. $\Omega_{n} \subset \subset$ $\Omega_{n+1}, \bigcup \Omega_{n}=\Omega$ ). If $f \in C^{\infty}(\Omega)$, we put

$$
\mu_{n}(f):=\sup \left\{\left|\partial^{\alpha} f(t)\right|: t \in \bar{\Omega}_{n},|\alpha| \leq n\right\}
$$

The ring of moderate nets of $C^{\infty}(\Omega)$ will be denoted $\mathcal{E}_{M}(\Omega)$.

The ideal of negligible nets will be denoted $\mathcal{N}(\Omega)$. The Colombeau extension $\mathcal{G}\left[C^{\infty}(\Omega)\right]$ will be denoted $\mathcal{G}(\Omega)$ and will be called the algebra of Colombeau generalized functions on $\Omega$. By the functoriality properties of Colombeau constructions, one immediately proves that

Proposition $1.4 \mathcal{G}(\Omega)$ is a differential topological algebra on $\overline{\mathbb{K}}$ and all differentiations are continuous mappings.

One defines in an obvious way the restriction of a generalized function on an open subset $\Omega^{\prime}$ of $\Omega$.

In fact we define a sheaf of topological algebras on $\overline{\mathbb{K}}$ which can be proved to be fine [15].

The support of a generalized function $f$ is the complement of the largest open subset $\Omega^{\prime}$ such that $f_{/ \Omega^{\prime}}=0$.

Clearly $\overline{\mathbb{K}}$ is trivially embedded in a subring of $\mathcal{G}(\Omega)$, the subring of 'constant generalized functions'.

A very important property of $\mathcal{G}(\Omega)$ is the fact that there exist embeddings of $\mathcal{D}^{\prime}(\Omega)$ into $\mathcal{G}(\Omega)$ :

Let $\phi \in \mathcal{S}\left(\mathbb{R}^{n}\right)$ (Schwartz functions) be such that $\int \phi d x=1$ and $\forall \alpha,|\alpha| \leq 1$ $\int x^{\alpha} \phi(x) d x=0$. For any $\left.\left.\varepsilon \in\right] 0,1\right]$, define the function $\phi_{\varepsilon}(x):=\frac{1}{\varepsilon^{n}} \phi\left(\frac{x}{\varepsilon}\right)$. If $T \in$ $\mathcal{D}^{\prime}(\Omega)$ has compact support, consider $i_{\phi}(T)=\left[\left(T * \phi_{\varepsilon}\right)_{\varepsilon}\right]$. By sheaf considerations and partition of unity this mapping can be extended on all $\mathcal{D}^{\prime}(\Omega)$. Such mappings respect derivations and products of $C^{\infty}$ functions but not the products of continuous 
functions (this would be impossible as is stated by a well known impossibility Theorem of L. Schwartz).

However the situation is not completely hopeless because we have in Colombeau theory some useful 'weak-equalities' (which are not stable under products):

(a) If $F=\left[\left(f_{\varepsilon}\right)\right]$ and $G=\left[\left(g_{\varepsilon}\right)\right], F$ and $G$ are said to be associated if

$$
\forall \rho \in \mathcal{D}(\Omega) \lim _{\varepsilon \rightarrow 0} \int\left(f_{\varepsilon}-g_{\varepsilon}\right) \rho d x=0
$$

(we denote $F \sim G$ ).

(b) $F$ and $G$ are said to be strongly associated (see [21,25]) which we denote $\left(F \sim_{S} G\right)$ if

$$
\forall \rho \in \mathcal{D}(\Omega), \exists a>0 \text { s.t. } \int\left(f_{\varepsilon}-g_{\varepsilon}\right) \rho d x=o\left(\varepsilon^{a}\right)
$$

(c) $F$ and $G$ are said to be 'equal in distribution sense' $\left(F \overline{\overline{\mathcal{D}^{\prime}}} G\right)$ if

$$
\forall \rho \in \mathcal{D}(\Omega) \int\left[f_{\varepsilon}-g_{\varepsilon}\right] \rho d x=0 .
$$

Oberguggenberger investigated many cases where, when the product of distributions $T, S$ is defined in some sense, then $i_{\phi}(T \cdot S)$ is 'weakly equal' to $i_{\phi}(T) i_{\phi}(S)$ in the sense of one of the above equivalence relations (see [23]).

The functorial structure of Colombeau constructions allows to define the value of a generalized function $f$ on a point $x_{0}$ of $\Omega$ as well as the integral of $f$ over a compact set $K$. Moreover it is clear that the mappings $f \rightarrow f\left(x_{0}\right)$ and $f \rightarrow \int_{K} f d x$ are continuous $\overline{\mathbb{K}}$ linear mappings for the respective sharp topologies.

Let us remind an important result proved using Landau inequality in [17].

Proposition 1.5 A net $\left(h_{\varepsilon}\right) \in \mathcal{E}_{M}(\Omega)$ is negligible $\left(\left(h_{\varepsilon}\right) \in \mathcal{N}(\Omega)\right)$ if and only if $\forall K \subset \subset \Omega, \forall a>0, \sup _{K}\left|h_{\varepsilon}\right|=o\left(\varepsilon^{a}\right)$.

Thus in this case we have to verify the rapid vanishing only of supremum seminorms and we need not evaluate the behaviour of derivatives.

The definition of negligible nets only by the evaluation of the supremum seminorms on relatively compact subsets allows us to prove easily the following.

Proposition 1.6 Let $E_{k}=C^{k}(\Omega)(k=1, \ldots, \infty)$.

There exist canonical mappings $j_{k}: \mathcal{G}\left[E_{k+1}\right] \rightarrow \mathcal{G}\left[E_{k}\right]$.

We denote $\mathcal{G}^{\prime}\left[E_{k}\right]:=j_{k}\left(\mathcal{G}\left[E_{k+1}\right]\right)$, with the relative topology of $\mathcal{G}\left[E_{k}\right]$.

Then $\mathcal{G}(\Omega)=\mathcal{G}\left[C^{\infty}(\Omega)\right]$ is embedded into a dense subset of $\mathcal{G}^{\prime}\left[E_{k}\right]$.

Proof The mappings are defined as the identity map on representatives. As $\mathcal{E}_{M}\left[E_{k+1}\right] \subset$ $\mathcal{E}_{M}\left[E_{k}\right]$ and $\mathcal{N}\left[E_{k+1}\right] \subset \mathcal{N}\left[E_{k}\right]$, the mapping $j_{k}$ is well-defined. 
Injectivity of the mapping $\mathcal{G}(\Omega) \rightarrow \mathcal{G}^{\prime}\left[E_{k}\right]$ is easily deduced by the fact that

$$
\mathcal{N}(\Omega)=\mathcal{E}_{M}(\Omega) \cap \mathcal{N}\left[E_{k}\right]
$$

In fact, by Proposition 1.5, the negligibility of the supremums on compact sets implies the negligibility of the supremums of all derivatives on the same sets. As for density, consider the modified nets $\delta_{n, \varepsilon}=\frac{1}{\varepsilon^{n d}} \phi\left(\frac{x}{\varepsilon^{n}}\right)$, where $\phi$ is a function whose fourier transform is a $C^{\infty}$ compactly supported function with value 1 on a neighbourhood of 0 . Let us now consider $\left(g_{\varepsilon}\right) \in \mathcal{E}_{M, c}\left(E_{k+1}\right)$ and $h_{\varepsilon}=g_{\varepsilon}-g_{\varepsilon} * \delta_{n, \varepsilon}$.

One can easily verify that $g_{\varepsilon} * \delta_{n, \varepsilon} \in \mathcal{E}_{M}(\Omega)$ and that $\forall K \subset \subset \Omega, \forall \alpha \in \mathbb{N}^{d},|\alpha| \leq$ $k, \forall a>0$

$$
\exists n_{0} \text { s.t. } n>n_{0} \Rightarrow \sup _{K}\left|\partial^{\alpha}\left(g_{\varepsilon}-g_{\varepsilon} * \delta_{n, \varepsilon}\right)\right|=o\left(\varepsilon^{a}\right) .
$$

\subsection{Generalized points}

J.F. Colombeau noticed that there exist non zero elements of $\mathcal{G}(\Omega)$ which take zero value on all points of $\Omega$. This led M. Kunzinger and M. Oberguggenberger to define the notions of 'generalized points' and 'compactly supported generalized points' (see [19]).

Definition 1.7 A net $\left(x_{\varepsilon}\right) \in \Omega^{] 0,1]}$ is said to be moderate if there exists $a \in$ $\mathbb{R}$ s.t. $\left\|x_{\varepsilon}\right\|=o\left(\varepsilon^{a}\right)$. Two such nets are said to be equivalent if

$$
\forall a>0,\left\|x_{\varepsilon}-y_{\varepsilon}\right\|=o\left(\varepsilon^{a}\right)
$$

The set of equivalence classes is called the set of generalized points and is denoted $\widehat{\Omega}$ and the class of such a net $\left(x_{\varepsilon}\right)$ is denoted as usually by $\left[\left(x_{\varepsilon}\right)\right]$. A generalized point $x=\left[\left(x_{\varepsilon}\right)\right]$ is said to be supported in a compact subset $K$ if there exists a representative $\left(x_{\varepsilon}^{\prime}\right)$ of $x$ such that $\forall \varepsilon \quad x_{\varepsilon}^{\prime} \in K$. The set of compactly supported generalized points is denoted $\widehat{\Omega}_{c}$.

Notice that if we put $\delta(x, y)=e^{-v\left(\left\|x_{\varepsilon}-y_{\varepsilon}\right\|\right)}$ (where $v$ is the valuation on $\overline{\mathbb{K}}$ ) then $\widehat{\Omega}_{c}$ acquires a complete ultrametric uniform structure. The topology thus defined is called the sharp topology of $\widehat{\Omega}_{c}$. Clearly $\Omega$ is embedded on a discrete subset of $\widehat{\Omega}_{c}$.

In some sense $\widehat{\Omega}$ is the 'Colombeau extension' of $\Omega$. By putting for $x \in \widehat{\Omega}_{c}$ and $f \in \mathcal{G}(\Omega), f(x)=\left[\left(f_{\varepsilon}\left(x_{\varepsilon}\right)\right)\right]$ we define the value of the generalized function $f\left(=\left[\left(f_{\varepsilon}\right)\right]\right)$ on the compactly supported generalized point $x=\left[\left(x_{\varepsilon}\right)\right]$.

One can easily verify that the mappings

$$
x \rightarrow f(x) \text { and } f \rightarrow f(x)
$$

are continuous for the respective sharp topologies. 
Remark If $x \in \widehat{\Omega}$ but $x \notin \widehat{\Omega}_{c}$, then $f(x)$ cannot always be defined. M. Kunzinger and M. Oberguggenberger proved in [19] that

Proposition 1.8 if $f \in \mathcal{G}(\Omega)$ and $\forall \xi \in \widehat{\Omega}_{c}, f(\xi)=0$ then $f=0$.

The notion of compactly supported generalized point allows us to obtain some properties analogous to those of continuous functions on $\Omega$.

Proposition 1.9 (Maximum theorem) Let $K$ be a compact subset of $\Omega$ and $f \in \mathcal{G}(\Omega)$ then there exists a generalized point $x_{0} \in \widehat{\Omega}_{c}$. s.t. $\forall y \in \widehat{\Omega}_{c}$, supported in $K|f(y)| \leq$ $\left|f\left(x_{0}\right)\right|$.

Proof Let $\left(x_{\varepsilon}\right) \in K$ be such that $\forall \varepsilon,\left|f_{\varepsilon}\left(x_{\varepsilon}\right)\right|=\sup _{K}\left|f_{\varepsilon}\right|$.

Clearly $\left[\left(x_{\varepsilon}\right)\right]$ satisfies the requirements of the proposition.

Proposition 1.10 Let $f$ be a 'real valued' generalized function on $\Omega$ (i.e. an element of $\mathcal{G}[E]$ where $E$ is the family of real valued $C^{\infty}$ functions). Let us suppose that $\Omega$ is arcwise connected and that $x$ and $y$ are two elements of $\widehat{\Omega}_{c}$ and $c \in \overline{\mathbb{R}}$ such that $f(x)<c<f(y)$. Then there exists $z=\left[\left(z_{\varepsilon}\right)\right] \in \widehat{\Omega}_{c}$ such that $f(z)=c$. (This proposition will be called the intermediate value theorem for generalized functions).

Proof Let $\Omega^{\prime}$ be a relatively compact connected subset of $\Omega$ such that both $x$ and $y$ are supported on some compact subset of $\Omega^{\prime}$. For $\varepsilon$ small enough, we have

$$
f_{\varepsilon}\left(x_{\varepsilon}\right) \leq c_{\varepsilon} \leq f_{\varepsilon}\left(y_{\varepsilon}\right)
$$

Let $z_{\varepsilon}$ be such that $f\left(z_{\varepsilon}\right)=c_{\varepsilon}$ and $z_{\varepsilon} \in \Omega^{\prime}$ (this is possible because $\Omega^{\prime}$ is connected and $f_{\varepsilon}$ is continuous). Clearly $z=\left[\left(z_{\varepsilon}\right)\right]$ satisfies the requirements of our proposition.

This simple property has important consequences concerning the set of maximal ideals of $\mathcal{G}(\Omega)$.

The notion of compactly supported generalized points allows us to have a 'generalized pointwise' criterium for invertibility (see also in [19]).

Proposition 1.11 A generalized function $f \in \mathcal{G}(\Omega)$ is invertible (as an element of the algebra $\mathcal{G}(\Omega)$ ) if and only if

$$
\forall \xi \in \widehat{\Omega}_{c}, f(\xi) \text { is invertible. }
$$

Let us remind a proof:

Proof Let us first remind that $x \in \overline{\mathbb{K}}$ is invertible if and only if there exists $a>0$ such that $|x|>\left[\left(\varepsilon^{a}\right)\right]$. Let $f$ be a generalized function.

Consider now a locally finite covering of $\Omega$ by relatively compact open subsets $\left(\omega_{i}, i \in I\right)$ and consider a $C^{\infty}$ partition of unity $\left(\varphi_{i}\right)$ adapted to this covering. On $\bar{\omega}_{i}$ consider $z_{\varepsilon}^{i}$ s.t. $\left|f_{\varepsilon}\left(z_{\varepsilon}^{i}\right)\right|=\inf _{\bar{\omega}_{i}}\left|f_{\varepsilon}(t)\right|$. By hypothesis if $z_{i}=\left[\left(z_{\varepsilon}^{i}\right)\right], f(z)$ is invertible, 
thus $\inf _{\omega_{i}}\left|f_{\varepsilon}(t)\right|>\varepsilon^{a}$ where $a$ is some positive constant. Clearly $\sup _{\omega_{i}} \frac{1}{|g(t)|}<\varepsilon^{-a}$, thus $g_{i} \in \mathcal{G}\left(\omega_{i}\right)$ defined by $g_{i}=\frac{1}{f(t)} \forall t \in \omega_{i}$ is such that $f_{/ \omega_{i}} g_{i}=1$. Consider now $G=\sum \varphi_{i} g_{i}$. Clearly $\varphi_{i} g_{i}$ define also an element of $\mathcal{G}(\Omega)$ and $G$ is well defined because the above sum is locally finite.

One verifies immediately that $G \cdot f=1$.

\subsection{Sequence model of Colombeau theory}

Another version of Colombeau extension is the sequence model where we simply replace a net $\left(f_{\varepsilon}\right)$ by a sequence $\left(f_{n}\right)$. The definition of moderateness or negligibility is simply what is obtained when we replace ' $\varepsilon$ ' by $\frac{1}{n}$. Clearly the notions of ' $\mathrm{n}$ valuations' and 'sharp' topology have obvious counterparts in the sequence model. This model was first used by the bielorussian group of Mathematicians around Radyno and Antonevich ([1]).

The sequence presentation was also used in [12] when authors introduced generalisations of the classical Colombeau constructions.

In some circumstances the sequence model allows a better understanding of the main points of a proof.

Such proofs can be translated in the net model in the following way. If the proposition is false there would exist a sequence $\left(f_{\varepsilon_{n}}\right)$ satisfying the negation of the proposition and this would give a counterexample in the sequence model.

We will also use the sequence model to present complicated counterexamples where the translation in the net model would just add useless difficulties of comprehension. Since our purpose here is to allow the reader to grasp the main ideas of our work we will freely pass from one model to the other.

Remark However arguments using cardinality must be treated with great caution because the two models don't have the same cardinal as sets.

\section{$1.6 m$-reduction}

In usual Colombeau theory the set of generalized constants is not a field but only a ring (contrary to what happens in the 'non standard' version of Colombeau theory (see $[20,23], \ldots)$.

However there is a shortcut to obtain in the frame of standard analysis algebras where the ring of constants is a field.

This procedure is the ' $m$-reduction' (see [27]) where $m$ is a maximal ideal of $\overline{\mathbb{K}}$. Those ' $m$-reduced' constructions are closely analogous to the non standard version of the theory see $[20,23,28]$ and are a direct rough way to construct something similar to ${ }^{\rho} \mathcal{E}(\Omega)$.

Definition 1.12 Let $m$ be a maximal ideal of $\overline{\mathbb{K}}$. The quotient field $\overline{\mathbb{K}}_{m}=\overline{\mathbb{K}} / m$ will be called the field of $m$-reduced generalized $\overline{\mathbb{K}}$-constants. The class of $x$ will be denoted $[x]_{m}$ and if $x$ is represented by $x_{\varepsilon}$, it will be denoted $\left[\left(x_{\varepsilon}\right)\right]_{m}$. 
In [2], the authors proved that any maximal ideal $m$ of $\overline{\mathbb{K}}$ is always closed for the sharp topology of $\overline{\mathbb{K}}$. Thus $\overline{\mathbb{K}}_{m}$ can be endowed with a quotient Hausdorff topology also called the sharp topology of $\overline{\mathbb{K}}_{m}$.

More precisely

Definition 1.13 If $x=\left[\left(x_{\varepsilon}\right)\right]_{m} \in \overline{\mathbb{K}}_{m}$ we define its valuation $v(x)$ by $v(x):=$ $\sup \left\{v\left(y_{\varepsilon}\right):\left[\left(y_{\varepsilon}-x_{\varepsilon}\right)\right] \in m\right\}$ and we define a distance $d(x, y):=e^{-v(x-y)}$ which is an ultrametric distance on $\overline{\mathbb{K}}_{m}$.

The topology and uniform structure thus defined are called the 'sharp' topology and uniform structure of $\overline{\mathbb{K}}_{m}$.

$\overline{\mathbb{K}}_{m}$ with its sharp uniform structure satisfies the following properties (see [27]).

Proposition 1.14 (a) If $\bar{m}=\overline{\mathbb{R}} \cap m$ then $\overline{\mathbb{C}}_{m}=\overline{\mathbb{R}}_{\bar{m}}+i \overline{\mathbb{R}}_{\bar{m}}$

(b) $v$ is a valuation i.e. $v$ satisfies also $v(x . y)=v(x)+v(y)$ (while in the case of $\overline{\mathbb{K}}$ we have only an inequality). $\overline{\mathbb{K}}_{m}$ is a complete ultrametric space.

(c) $\overline{\mathbb{R}}_{m}$ is totally ordered by the following relation $\widehat{x} \geq \widehat{y}$ which means by definition that there exist respective representing nets $\left(x_{\varepsilon}\right),\left(y_{\varepsilon}\right)$ s.t. $x_{\varepsilon} \geq y_{\varepsilon}$. The order topology coincides with the sharp topology.

(d) $\overline{\mathbb{R}}_{m}$ is a non archimedian, real closed field

(e) $\mathbb{K}$ is naturally embedded on a discrete subfield of $\overline{\mathbb{K}}_{m}$.

(f) $\overline{\mathbb{R}}_{m}$ is complete but not Dedekind complete (i.e. a bounded (for order) subset does not always admit an upper bound) .

(g) If $x \in \overline{\mathbb{R}}_{m}$ is limited i.e. $\exists N \in \mathbb{N}$ such that $-N<x<N$. Then there exists a unique element $x_{0}$ of $\mathbb{R}$ such that $\left|x-x_{0}\right|$ is smaller than every positive standard real number, $x_{0}$ will be called the shadow of $x$ and we will say that $x$ belongs to the 'halo' of $x_{0}$.

h) Then the halo of zero is a subring of $\overline{\mathbb{R}}_{m}$, both closed and open for sharp topology.

Here we will remind only the proof of $\mathrm{g}$ ). As $\overline{\mathbb{R}}_{m}$ is totally ordered, let $A$ be the set of classical reals bigger than $x$. $A$ is non empty and bounded since $x$ is limited. Let $x_{0}$ be the infimum of $A$. For any $n \in \mathbb{N},\left|x-x_{0}\right|<\frac{1}{n}$.

Notice that on $\overline{\mathbb{C}}_{m}$ we can define an 'absolute value' with value in $\overline{\mathbb{R}}_{m}^{+}$in the following way.

If $x=\left[\left(x_{\varepsilon}\right)\right]_{m}$, then $|x|:=\left[\left(\left|x_{\varepsilon}\right|\right)\right]_{m}$. This absolute value satisfies all classical properties.

We can now easily give the definition of the $m$-reduced Colombeau extension of $\left(E,\left(\mu_{n}\right)_{n}\right)($ see [27]).

Definition 1.15 Let $\left(E,\left(\mu_{n}\right)\right)$ be a topological vector space topologized by an increasing sequence of seminorms $\left(\mu_{n}\right)$.

An element $f=\left[\left(f_{\varepsilon}\right)\right]$ of $\mathcal{G}[E]$ is said to be $m$-negligible if and only if

$$
\left.\forall n \in \mathbb{N}\left[\mu_{n}\left(f_{\varepsilon}\right)\right)\right] \in m
$$

The subspace of $m$-negligible elements of $\mathcal{G}[E]$ will be denoted $J_{m}[E]$. The $m$-reduced Colombeau extension $\mathcal{G}_{m}[E]$ is defined as the factor space $\mathcal{G}_{m}[E]=$ 
$\mathcal{G}[E] / J_{m}[E]$ by putting $v_{n}(f):=\sup \left\{v_{n}(g): g\right.$ represents $\left.f\right\}$ and

$$
\delta_{n}(f, g)=e^{-v_{n}(f, g)}
$$

We define on $\mathcal{G}_{m}[E]$ a complete ultrametric Hausdorff uniform structure called the sharp uniform structure and a Hausdorff topology called the sharp topology of $\mathcal{G}_{m}[E]$.

Using the definitions we can prove the following results (the proof is cumbersome but straightforward)

Proposition $1.16 \mathcal{G}_{m}[E]$ is a topological vector space on the topological field $\overline{\mathbb{K}}_{m}(\mathbb{K}$ being the field of real or complex numbers according to the case we are dealing with); and when $E$ is a topological algebra over $\mathbb{K}$, then $\mathcal{G}_{m}[E]$ is a topological algebra over $\overline{\mathbb{K}}_{m}$. More generally, if $L$ is a continuous linear mapping from $E$ to $F$ then it defines canonically a $\overline{\mathbb{K}}_{m}$-linear continuous mapping (also denoted $L$ ) from $\mathcal{G}_{m}[E]$ to $\mathcal{G}_{m}[F]$. If $\varphi$ is a continuously tempered mapping (see [9] and [27]) from $E$ to $F$, then it defines a continuous mapping from $\mathcal{G}_{m}[E]$ to $\mathcal{G}_{m}[F]$.

Thus the $m$-reduction is also a 'Functorial' procedure (see [27]).

\section{$1.7 m$-reduced generalized points}

The above ' $m$-reduction' procedure can also be applied on the case of the set of generalized points:

Definition 1.17 Two generalized points $(x, y)=\left(\left[\left(x_{\varepsilon}\right)\right],\left[\left(y_{\varepsilon}\right)\right]\right)$ are said to be $m$-equivalent if $\left[\left(\left\|x_{\varepsilon}-y_{\varepsilon}\right\|\right)\right] \in m$. The set of equivalence classes thus obtained is denoted $\widehat{\Omega}_{m}$. The set of classes admitting a compactly supported representative will be denoted $\widehat{\Omega}_{m, c}$ and will be called the set of compactly supported $m$-reduced generalized points. The sharp topology is defined also on $\widehat{\Omega}_{m, c}$ by the same procedures as previously.

An important property of $\widehat{\Omega}_{m, c}$ (whose counterpart is not valid for $\widehat{\Omega}_{c}$ ) is the following.

Proposition 1.18 If $\widehat{x} \in \widehat{\Omega}_{m, c}$ then there exists a unique element $x_{0}$ of $\Omega$ s.t. $\left\|\widehat{x}-x_{0}\right\|<$ $1 / n, \forall n \in \mathbb{N}$. Such a point will be called the shadow of $\widehat{x}$.

The set of elements of $\widehat{\Omega}_{m, c}$ admitting $x_{0}$ as a shadow is called the halo of $x_{0}$.

Proof If $\widehat{\Omega}_{m, c} \subset\left(\mathbb{R}_{m}\right)^{n}$ the sentence can be easily proved using proposition $1.14 \mathrm{~g}$ ) for any given coordinate thus for the whole space $\left(\mathbb{R}_{m}\right)^{n}$, i.e. one can prove that all limited elements of $\left(\mathbb{R}_{m}\right)^{n}$ admit such a shadow and all elements of $\widehat{\Omega}_{m, c}$ are limited. More over it is obvious that their shadow lies in $\Omega$.

In some sense $\widehat{\Omega}_{m, c}$ is some kind of a 'nonstandard' extension of $\Omega$.

As in the case of $\mathcal{G}_{m}[E]$ we can define on $\widehat{\Omega}_{m, c}$ a 'sharp' topology which coincides with the topology given by the $\overline{\mathbb{R}}_{m}^{+}$-valued 'norm' $\|x-y\|=\left[\left(\left\|x_{\varepsilon}-y_{\varepsilon}\right\|\right)\right]_{m}$.

This topology can be given also by an ultrametric distance (see [27]). 


\subsection{The algebra $\mathcal{G}_{m}(\Omega)$}

Starting from $E=C^{\infty}(\Omega)$ with its set of seminorms $\mu_{n}$ we obtain the algebra $\mathcal{G}_{m}(\Omega)$ of $m$-reduced generalized functions (for details see [27]). In this case the subspace of $m$-negligible elements will be denoted $\mathcal{J}_{m}(\Omega)$.

Using the same method as M. Kunzinger and M. Oberguggenberger in [19] and the fact that all ideals of $\overline{\mathbb{K}}$ are convex (i.e. if $|x| \leq|y|$ and $|y|$ belongs to the ideal, so does $x$ ) we obtain the following important remark.

Proposition $1.19 f=\left[\left(f_{\varepsilon}\right)\right]$ belongs to $\mathcal{J}_{m}(\Omega)$ if and only if for any compact subset $K$ of $\Omega, \quad\left[\sup _{K}\left|f_{\varepsilon}\right|\right] \in m$.

We give the proof in the simplified case of one variable. By multiplying $f_{\varepsilon}$ by a $C^{\infty}$ compactly supported generalized function $\varphi$, we can without loss of generality (evaluation been always done on compact spaces) suppose that $f_{\varepsilon} \varphi=g_{\varepsilon}$ has moderate uniform evaluations for all derivatives. Let us remind the Landau inequalities : if $M_{0}$ is the maximum of a function $f, M_{1}$ the maximum of its derivatives and $M_{2}$ the maximum of its second derivatives, then $M_{1} \leq \sqrt{2 M_{0} \cdot M_{2}}$. Let $M_{0, \varepsilon} ; M_{1, \varepsilon} ; M_{2, \varepsilon}$ be the corresponding maximums for the function $g_{\varepsilon}$, then we will prove that if $\left[\left(M_{0, \varepsilon}\right)\right] \in m$, then also $\left[\left(M_{1, \varepsilon}\right)\right] \in m$. The above mentioned inequality implies that $M_{1, \varepsilon}^{2} \leq 2 M_{0, \varepsilon} \cdot M_{2, \varepsilon}$ and as $\left[\left(M_{0, \varepsilon}\right)\right] \in m$ and $\left[\left(M_{2, \varepsilon}\right)\right] \in \overline{\mathbb{R}}$, we have that $\left[\left(M_{1, \varepsilon}\right)^{2}\right]$ belongs to $m$. But as $m$ is a maximal ideal, we can conclude that also $\left[\left(M_{1, \varepsilon}\right)\right]$ belongs to $m$ since it is a prime ideal. By induction we can prove that, for all orders, the bounds of derivatives belong to $m$, which implies that $\left(f_{\varepsilon}\right) \in \mathcal{J}_{m}(\Omega)$.

One can easily verify that, as in the case of $\mathcal{G}(\Omega)$, we define by this procedure a sheaf of algebras and a notion of restriction to open subsets.

Let $1_{\theta}$ be an embedding of $\mathcal{D}^{\prime}(\Omega)$ into $\mathcal{G}(\Omega)$. If for any $T \in \mathcal{D}^{\prime}(\Omega)$ we associate its image $\left[1_{\theta}(T)\right]_{m}$ into $\mathcal{G}_{m}(\Omega)$, we obtain an embedding of distributions into the algebra $\mathcal{G}_{m}(\Omega)$ respecting derivations of distributions and products of $C^{\infty}$ functions. But this time the 'constants' of our algebra form a field and not just a ring as in the case of $\mathcal{G}(\Omega)$.

Clearly $\overline{\mathbb{K}}_{m}$ is embedded in an obvious way into $\mathcal{G}_{m}(\Omega)$ and its elements can be considered 'constant' generalized $m$-reduced functions.

As in the case of $\mathcal{G}(\Omega)$ we can evaluate $m$-reduced generalized functions on $m$-reduced compactly supported generalized points.

Proposition-definition If $f=\left[\left(f_{\varepsilon}\right)\right]_{m} \in \mathcal{G}_{m}(\Omega)$ and $x=\left[\left(x_{\varepsilon}\right)\right]_{m} \in \widehat{\Omega}_{m, c}$ then $\left[\left(f_{\varepsilon}\left(x_{\varepsilon}\right)\right)\right]_{m}$ does not depend on the choice of representatives $\left(f_{\varepsilon}\right)$ and $\left(x_{\varepsilon}\right)$. This $m$-reduced generalized constant will be called the value of $f$ on $x,(f(x))$. Moreover the mappings $f \rightarrow f(x)$ and $x \rightarrow f(x)$ are continuous for the respective sharp topologies.

The proof of this proposition is straightforward (see [25]) and is an easy consequence of the 'functoriality' of $m$-reduction. One can also easily prove that $\mathcal{G}_{m}(\Omega)$ is a topological differential algebra for a fixed $\overline{\mathbb{K}}_{m}$ where $\mathbb{K}=\mathbb{R}$ or $\mathbb{C}$.

If $\phi: \mathbb{C} \rightarrow \mathbb{C}$ is an element of $\mathcal{O}_{M}(\mathbb{C})$ and if $g \in \mathcal{G}_{m}(\Omega)$, we can define $\phi \circ g$ :

Proposition 1.20 If $g \in \mathcal{G}_{m}(\Omega), g=\left[\left(g_{\varepsilon}\right)\right]_{m}$ and $\phi \in \mathcal{O}_{M}$. Then $\left[\left(\phi\left(g_{\varepsilon}\right)\right)\right]_{m}$ does not depend on the choice of representatives. It will be denoted $\phi(g)$ or $\phi \circ g$. 
Proof Let $\left(\widehat{g}_{\varepsilon}\right)$ be another representative. For any convex compact subset $K$ of $\Omega$, there exists a temperate mapping $\varepsilon \rightarrow R_{\varepsilon} \leq \frac{C}{\varepsilon^{N}} \quad(N, C$, being adequate constants $)$ such that for any $\varepsilon$ and any $x \in K$ both $g_{\varepsilon}(x)$ and $\widehat{g}_{\varepsilon}(x)$ belong to $B\left(0, R_{\varepsilon}\right)$. Thus

$$
\sup _{x \in K}\left(\left|\phi\left(g_{\varepsilon}(x)\right)-\phi\left(\widehat{g}_{\varepsilon}(x)\right)\right|\right) \leq \sup _{B\left(0, R_{\varepsilon}\right)}|\nabla \phi| \sup _{K}\left|g_{\varepsilon}-\widehat{g}_{\varepsilon}\right|
$$

and by hypothesis, as $\phi$ is in $\mathcal{O}_{M}$ and the increase of $\left(R_{\varepsilon}\right)$ is moderate, and $\left[\left(\left|g_{\varepsilon}-\widehat{g}_{\varepsilon}\right|\right)\right]$ belongs to $\mathcal{J}_{m}(\Omega)$,we can conclude that $\left[\sup \left|g_{\varepsilon}-\widehat{g}_{\varepsilon}\right|\right] \in m$. Thus this is also the case for

$$
\left.\underset{K}{\left[\sup _{K}\right.}\left|\phi\left(g_{\varepsilon}(x)\right)-g\left(\widehat{g}_{\varepsilon}(x)\right)\right|\right]_{m}
$$

hence $\left[\phi\left(g_{\varepsilon}\right)-\phi\left(\widehat{g}_{\varepsilon}\right)\right] \in \mathcal{J}_{m}(\Omega)$. As any compact set is included in a finite union of balls, the proof is complete.

Proposition 1.21 Maximum theorem.

If $f \in \mathcal{G}_{m}(\Omega)$ and $\Omega^{\prime} \subseteq \subset \Omega$ is a relatively compact subset of $\Omega$ then there exists $x_{0} \in \widehat{\Omega}_{m, c}$, supported in $\overline{\Omega^{\prime}}$ s.t.

$$
\forall y \in \widehat{\Omega}_{m, c}, \quad y \text { supported in } \overline{\Omega^{\prime}} \text { then }|f(y)| \leq\left|f\left(x_{0}\right)\right| .
$$

Proof Let $\left[\left(f_{\varepsilon}\right)\right]$ represent $f$ and let $x_{\varepsilon}$ be a point on which is attained the maximum of the function $f_{\varepsilon}$ on $\overline{\Omega^{\prime}}$. The class of the net $\left(x_{\varepsilon}\right)$ is supported in $\overline{\Omega^{\prime}}$. Let $y$ be any $m$-reduced generalized point supported in $\overline{\Omega^{\prime}}$. By definition it admits a representative $\left(y_{\varepsilon}\right)$ supported in $\overline{\Omega^{\prime}}$, and hence $\left|f_{\varepsilon}\left(y_{\varepsilon}\right)\right| \leq\left|f_{\varepsilon}\left(x_{\varepsilon}\right)\right|$ which implies, passing to $m$-reduction, that $|f(y)| \leq|f(x)|$.

Now we can easily establish the characterization of $m$-reduced generalized functions by their values on $m$-reduced generalized points, which amounts to the following proposition:

Proposition 1.22 If $\forall x \in \widehat{\Omega}_{m, c} f(x)=0$, then $f=0$.

This is an easy consequence of the previous proposition.

Proposition 1.23 (Intermediate value theorem) Let $\Omega$ be an open connected subset of $\mathbb{R}^{n}$ and $f$ a 'real' $m$-reduced generalized function. Let us suppose that $x$ and $y$ are two elements of $\widehat{\Omega}_{m, c}$ and $c \in \overline{\mathbb{K}}_{m}$ s.t. $f(x)<c<f(y)$. Then there exists $z \in \widehat{\Omega}_{m, c}$ s.t. $f(z)=c$.

Proof For simplicity without loss of generality we suppose that $\Omega$ is convex (going to the general case is straightforward but cumbersome). The hypothesis implies that there exist representing nets $\left(f_{1, \varepsilon}\right)$ and $\left(f_{2, \varepsilon}\right)$ of $f,\left(x_{\varepsilon}\right)$ representing $x$ and $\left(y_{\varepsilon}\right)$ representing $y$ and $\left(c_{\varepsilon}\right)$ representing $c$ s.t.

$$
f_{1, \varepsilon}\left(x_{\varepsilon}\right) \leq c_{\varepsilon} \leq f_{2, \varepsilon}\left(y_{\varepsilon}\right) \text { for } \varepsilon \text { small enough. }
$$


Since $|y-x|$ is a non zero element of $\overline{\mathbb{K}}_{m}$, it is an invertible element of $\overline{\mathbb{K}}_{m}$. Thus $|y-x|$ has a positive inverse which is majorated by $\varepsilon^{-a}$, where $a$ is some positive real constant. We can conclude that $|y-x|$ is minorated by $\varepsilon^{a}$ which implies that it has an invertible representative. For fixed representatives of $x$ and $y$, we can freely choose $f_{1, \varepsilon}$ and $f_{2, \varepsilon}$ satisfying the above conditions. Let $\Omega^{\prime}$ be a convex relatively compact subset of $\Omega$ s.t. $\left(x_{\varepsilon}\right)$ and $\left(y_{\varepsilon}\right)$ are both supported in it. Consider now a net $\left(\ell_{\varepsilon}\right)$ of affine functions s.t.

$$
\ell_{\varepsilon}\left(x_{\varepsilon}\right)=0, \ell_{\varepsilon}\left(y_{\varepsilon}\right)=f_{2, \varepsilon}\left(y_{\varepsilon}\right)-f_{1, \varepsilon}\left(y_{\varepsilon}\right),
$$

By invertibility of $\left|\left(y_{\varepsilon}-x_{\varepsilon}\right)_{\varepsilon}\right|$ the slope of $\ell_{\varepsilon}$ is clearly bounded by a moderate multiple of

$$
\sup _{\Omega^{\prime}}\left|f_{2, \varepsilon}-f_{1, \varepsilon}\right| \text {. }
$$

Thus $\left[\left(\ell_{\varepsilon}\right)\right]$ belongs to $\mathcal{J}_{m}(\Omega)$; hence $\left(f_{1, \varepsilon}+l_{\varepsilon}\right)=\left(\tilde{f}_{\varepsilon}\right)$ is another net representing $f$. This net satisfies

$$
\tilde{f}_{\varepsilon}\left(x_{\varepsilon}\right) \leq c_{\varepsilon} \leq \tilde{f}_{\varepsilon}\left(y_{\varepsilon}\right)
$$

and hence $\Omega^{\prime}$ being convex there exists $z_{\varepsilon} \in \Omega^{\prime}$ s.t. $f\left(z_{\varepsilon}\right)=c_{\varepsilon}$. Clearly $z=\left[\left(z_{\varepsilon}\right)\right]_{m}$ satisfies the requirements of the proposition.

When an $m$-reduced generalized function takes non zero values on all elements of $\left(\widehat{\Omega}_{m, c}\right)$, it turns out that it is invertible (contrarily to what happens in the non $m$-reduced setting where we have to suppose that all values are invertible).

Proposition $1.24 f \in \mathcal{G}_{m}(\Omega)$ is invertible if and only if

$$
\forall x \in \widehat{\Omega}_{m, c} f(x) \neq 0 .
$$

Proof Necessity is obvious. Let us now suppose that $\forall x \in \widehat{\Omega}_{m, c}, \quad f(x) \neq 0$. Let $\Omega^{\prime} \subset \subset \Omega$ be a relatively compact open subset of $\Omega$ and $\left(f_{\varepsilon}\right)_{\varepsilon}$ be a net representing $f$. There exists for any $\varepsilon$ an element $x_{\varepsilon}$ of $\overline{\Omega^{\prime}}$ such that $f_{\varepsilon}\left(x_{\varepsilon}\right)=\inf _{\Omega^{\prime}}\left|f_{\varepsilon}\right|$. Our hypothesis amounts to saying that there exists $b>0$ and $\left[a_{\varepsilon}\right] \in m$ s.t. $\left|f_{\varepsilon}+a_{\varepsilon}\right|^{2}>\varepsilon^{2 b}$ on $\Omega^{\prime}$.

Notice that $f_{1, \varepsilon}=\left(f_{\varepsilon}+a_{\varepsilon}\right) / \Omega^{\prime}$ is another representative of $f$. Consider now $h_{\varepsilon}=\frac{\overline{f_{1, \varepsilon}}}{\left|f_{\varepsilon}+a_{\varepsilon}\right|^{2}}$. The net $\left(h_{\varepsilon}\right)$ is moderate on $\Omega^{\prime}$; moreover $f_{1, \varepsilon} \cdot h_{\varepsilon}=1$. Hence if $h \in \mathcal{G}_{m}\left(\Omega^{\prime}\right), h=\left[\left(h_{\varepsilon}\right)\right]_{m}$, it is clear that $f_{/ \Omega^{\prime}} \cdot h=1$.

Let us now consider a locally finite covering of $\Omega^{\prime}$ by relatively compact open subsets $\left(\omega_{i, i \in I}\right)$ and construct $h_{i} \in \mathcal{G}_{m}\left(\omega_{i}\right)$ as above. Consider also a $C^{\infty}$ - partition of unity $\left(\varphi_{i}\right)_{i \in I}$ adapted to the covering $\left(\omega_{i}\right)_{i \in I}$. Then $\varphi_{i} h_{i}$ has a compact support included in $\omega_{i}$ and thus defines also an element of $\mathcal{G}_{m}(\Omega)$. The partition being locally finite we can define $H=\sum \varphi_{i} h_{i} \in \mathcal{G}_{m}(\Omega)$. Now we clearly have: $f H=\sum \varphi_{i} h_{i} f=$ $\sum \varphi_{i}=1$. 


\section{Preliminaries on ideals of $\mathcal{G}(\Omega)$}

\section{1 'Localization' of ideals}

We saw that the structure of generalized function has two aspects: they are a sheaf of algebras and they define continuous mapping from the set of compactly supported generalized points into the ring of generalized constants. Let us first give a notion linked to the 'sheaf theoretic' aspect of the theory.

Let $\mathcal{J} \subset \mathcal{G}(\Omega)$ be an ideal of $\mathcal{G}(\Omega)$. If $\Omega^{\prime} \subset \Omega$ is an open subset of $\Omega$ it might happen that $\mathcal{J}_{/} \Omega^{\prime}$ contains all generalized constants in which case it spans $\mathcal{G}\left(\Omega^{\prime}\right)$, i.e. it is locally 'irrelevant'.

Thus studying an ideal $\mathcal{J}$ it is relevant to know 'where' it does not span everything. This leads to the following notion already investigated in [2].

Definition 2.1 A point $x_{0} \in \Omega$ is said to be 'regular' for an ideal $\mathcal{J}$ of $\mathcal{G}(\Omega)$ if there exists an open neighborhood $\omega$ of $x_{0}$ in $\Omega$ such that $\mathcal{J} / \omega$ spans $\mathcal{G}(\omega)$. Clearly the set of all points of $\Omega$ which are 'regular' for the ideal $\mathcal{J}$ is an open subset of $\Omega$. Its complement is called the support of $\mathcal{J}$ (or the irregular support of $\mathcal{J}$ ).

Example The ideal spanned by $[(x-\varepsilon)]$ in $\mathcal{G}(\mathbb{R})$ has $\{0\}$ as support. Because if $x_{0} \neq 0$ there is a neighborhood $\omega$ of $x_{0}$ on which $[(x-\varepsilon)]$ is invertible; while it is not invertible on any neighborhood of 0 . Concerning support of ideals we have the following properties, already proved in [2].

Proposition 2.2 (a) If $\mathcal{J}_{1} \subset \mathcal{J}_{2}$ are two proper ideals of $\mathcal{G}(\Omega)$, then

$$
\operatorname{supp}\left(\mathcal{J}_{2}\right) \subset \operatorname{supp}\left(\mathcal{J}_{1}\right)
$$

(b) If $\mathcal{J}$ is a proper ideal of $\mathcal{G}(\Omega)$, then $\mathcal{J}$ is dense in $\mathcal{G}(\Omega)$ if and only if $\operatorname{supp}(\mathcal{J})=\emptyset$.

(c) If $\mathcal{J}$ is a prime ideal of $\mathcal{G}(\Omega)$ then $\operatorname{card}(\operatorname{supp}(\mathcal{J})) \leq 1$.

We will give a rapid sketch of the proof (for more details see [2]).

Proof (a) If $\mathcal{J}_{1} / \omega$ contains 1 , so does $\mathcal{J}_{2} / \omega$.

(b) $\mathcal{J}$ is dense if and only if for any relatively compact open subset $\omega$ there exists $f \in \mathcal{J} \quad f=\left[\left(f_{\varepsilon}\right)\right]$ such that for $\varepsilon$ small enough $\sup _{\omega}\left|1-f_{\varepsilon}\right|<\frac{1}{2}$. Thus $f / \omega$ is invertible (because $\left|f_{\varepsilon}\right|>\frac{1}{2}$ ) and hence no point is irregular. Conversely. Let us suppose that $\operatorname{supp}(\mathcal{J})=\varnothing$. By hypothesis for any $x \in \Omega$ there exists an open neighborhood $\omega_{x}$ and $f_{x} \in \mathcal{J}$ such that $f_{x} / \omega_{x}=1$. Let $\Omega^{\prime}$ be an open relatively compact subset of $\Omega$. $\overline{\Omega^{\prime}}$ being compact there exists a finite subcovering $\left(\omega_{a_{n}}\right)$ of $\overline{\Omega^{\prime}}$ and a $C^{\infty}$ partition of unity $\varphi_{n}$ adapted to this subcovering. Clearly $\sum \varphi_{n} f_{n} / \Omega^{\prime}=1$. Thus $\mathcal{J}$ is dense.

(c) Let us suppose that $\operatorname{card}(\operatorname{supp}(\mathcal{J}))>1$. Then there would exist two points $x_{1}$ and $x_{2}$ of $\Omega$, both belonging to $\operatorname{supp}(\mathcal{J})$ and $x_{1} \neq x_{2}$.

Let $\varphi_{1}, \varphi_{2}$ be two elements of $\mathcal{G}(\Omega)$ such that $\operatorname{supp}\left(\varphi_{1}\right) \cap \operatorname{supp}\left(\varphi_{2}\right)=\emptyset$ and such that there exist neighborhoods $\omega_{1}$ and $\omega_{2}$ of $x_{1}$ and $x_{2}$ respectively such that $\varphi_{1} / \omega_{1}=1$ 
and $\varphi_{2} / \omega_{2}=1$. Since $\varphi_{1} \cdot \varphi_{2}=0 \in \mathcal{J}, \varphi_{1}$ or $\varphi_{2}$ has to be in $\mathcal{J}$ ( $\mathcal{J}$ is prime). Thus $x_{1}$ or $x_{2}$ has to be regular for $\mathcal{J}$.

Remark The proof of (b) has the following consequence (see [2]).

Corollary $2.3 \operatorname{Let}_{\mathcal{C}}(\Omega)$ be the set of the compactly supported elements of $\mathcal{G}(\Omega)$. $\mathcal{G}_{c}(\Omega)$ is the smallest dense ideal of $\mathcal{G}(\Omega)$.

Remark Clearly different ideals can have the same support. For example, the ideal $\mathcal{J}_{1}$ spanned by $[(x-\varepsilon)]$ and the ideal $\mathcal{J}_{2}$ spanned by $[(x)]$ have both $\{0\}$ as support.

\subsection{Generalized trace of ideals}

As generalized functions define continuous mappings of $\widehat{\Omega}_{c}$ into $\overline{\mathbb{K}}$, we can define $\mathcal{J}(\xi):=\{f(\xi): f \in \mathcal{J}\}$ for $\xi \in \widehat{\Omega}_{c}$ and $\mathcal{J}$ an ideal of $\mathcal{G}(\Omega)$. It is clear that $\mathcal{J}(\xi)$ is an ideal of $\overline{\mathbb{K}}$.

Thus it is relevant to know on which $\xi \in \widehat{\Omega}_{c}, \mathcal{J}(\xi)$ is a proper ideal:

Definition 2.4 If $\mathcal{J}$ is a proper ideal of $\mathcal{G}(\Omega)$ we define the generalized trace of $\mathcal{J}(\operatorname{Tr}(\mathcal{J}))$ by

$$
\operatorname{Tr}(\mathcal{J}):=\left\{\xi \in \widehat{\Omega}_{c} \text { s.t. } \mathcal{J}(\xi) \neq \overline{\mathbb{K}}\right\}
$$

Example The ideal $\mathcal{J}$ of $\mathcal{G}(\mathbb{R})$ spanned by $\left[\left(x-\frac{1}{\varepsilon}\right)\right]$ is such that $\operatorname{Tr}(\mathcal{J})=$ $\left\{x\right.$ such that $\left[\left(x_{\varepsilon}-\frac{1}{\varepsilon}\right)_{\varepsilon}\right]$ is not invertible $\}$

Remark One might be led to suppose that if $\xi \in \operatorname{Tr}(\mathcal{J})$, then there exists $x \in \operatorname{supp}(\mathcal{J})$ s.t. $\left(\xi_{\varepsilon}\right) \rightarrow x$. This is not always the case. For example, if $\mathcal{J}$ is spanned by $\left[\left(x-\sin \left(\frac{1}{\varepsilon}\right)\right]\right.$, its generalized trace contains $\xi=\left[\left(\sin \frac{1}{\varepsilon}\right)\right]$ but $\sin \frac{1}{\varepsilon}$ admits no limit when $\varepsilon$ tends to zero. In this example it is easy to show that the support consists in the whole interval $[-1,1]$.

However it is clear that when $\operatorname{supp}(\mathcal{J})=\emptyset$, then $\operatorname{Tr}(\mathcal{J})=\emptyset$.

An easy way of making ideals is the following.

Definition 2.5 Let $A \subset \widehat{\Omega}_{c}$ and $a$ be a proper ideal of $\overline{\mathbb{K}}$.

$\mathcal{G}_{A, a}(\Omega)$ is the ideal constituted of all elements $f \in \mathcal{G}(\Omega)$ s.t.

$$
\forall \xi \in A \quad f(\xi) \in a
$$

(see [2]).

Generalized traces have the following properties (see [2]):

Proposition 2.6 (a) If $\mathcal{J}_{1} \subseteq \mathcal{J}_{2}$ are two proper ideals of $\mathcal{G}(\Omega)$, then $\operatorname{Tr}\left(\mathcal{J}_{2}\right) \subseteq \operatorname{Tr}\left(\mathcal{J}_{1}\right)$. 
(b) Let $\Omega$ be convex. If $\left|x-x_{0}\right| \in a$, then $x \in \operatorname{Tr}\left(\mathcal{G}_{x_{0}, a}(\Omega)\right)$. More generally the trace of $\mathcal{G}_{A, a}$ consists of all elements $y \in \widehat{\Omega}_{c}$ s.t. there exists $\xi \in A$ s.t. $\|y-\xi\| \in a$.

Proof (a) It is straightforward using the definition of generalized trace.

(b) Let $K$ be a convex compact subset of $\Omega$ in which both $y$ and $\xi$ are compactly supported then

$$
\left|f_{\varepsilon}\left(y_{\varepsilon}\right)-f_{\varepsilon}\left(\xi_{\varepsilon}\right)\right| \leq \sup _{K}\left|f_{\varepsilon}^{\prime}\right| \cdot\left\|y_{\varepsilon}-\xi_{\varepsilon}\right\|
$$

thus there exists a positive generalized constant $R$ s.t.

$$
\left[\mid\left(f_{\varepsilon}\left(y_{\varepsilon}\right)-f_{\varepsilon}\left(\xi_{\varepsilon}\right) \mid\right] \leq R\left[\left\|y_{\varepsilon}-\xi_{\varepsilon}\right\|\right]=R\|y-\xi\|\right.
$$

As ideals of $\overline{\mathbb{K}}$ are convex this implies that $\left[\left(f_{\varepsilon}(y)-f_{\varepsilon}\left(\xi_{\varepsilon}\right)\right)\right] \in a$ and hence $f(y) \in a$.

One can also easily verify the following (see [2]):

Proposition 2.7 (a) If a is a prime ideal of $\overline{\mathbb{K}}$ and $\xi \in \widehat{\Omega}_{c}$, then $\mathcal{G}_{\xi, a}(\Omega)$ is a prime ideal of $\mathcal{G}(\Omega)$.

(b) If $m$ is a maximal ideal of $\overline{\mathbb{K}}$, then $\mathcal{G}_{\xi, m}(\Omega)$ is a maximal ideal of $\mathcal{G}(\Omega)$ and

$$
\operatorname{Tr}\left(\mathcal{G}_{\xi, m}(\Omega)\right)=\left\{\xi^{\prime} \in \Omega_{c} \text { s.t. }\left|\xi^{\prime}-\xi\right| \in m\right\}
$$

If an ideal $\mathcal{M}$ of $\mathcal{G}(\Omega)$ is a maximal ideal then either it is dense, in which case its support and trace are void, or it is closed, in which case its support is exactly one point of $\Omega$.

One might hope that all closed maximal ideals of $\mathcal{G}(\Omega)$ are of the form $\mathcal{G}_{\xi, m}(\Omega)$ where $\xi \in \widehat{\Omega}_{c}$ and $m$ is a maximal ideal of $\overline{\mathbb{K}}$.

If all closed maximal ideals were of this form, we would at least have finished with the characterisation of closed maximal ideals of $\mathcal{G}(\Omega)$ (they would be in a one to one correspondence with couples $(\xi, m)$ where $\xi \in \widehat{\Omega}_{c}$ and $m$ maximal ideal of $\overline{\mathbb{K}}$ more or less in the same way that the maximal ideals of $C(K)$ where $K$ is compact are in correspondence with elements of $K$ ).

Unfortunately this is not the case (see [2]).

Proposition 2.8 There exist closed maximal ideals which are not of the form $\mathcal{G}_{\xi, m}(\Omega)$.

Proof Let $x_{0}$ be an element of $\Omega$ and $\mathcal{J}_{1}$ the set of all generalized functions $f$ such that $x_{0} \notin \operatorname{supp}(f)$.

Let $\mathcal{J}_{2}$ be the ideal spanned by $\mathcal{J}_{1}$ and the following subset $\mathcal{A}$ of $\mathcal{G}(\Omega)$

$\mathcal{A}=\left\{\varphi \in \mathcal{G}(\Omega): \exists \xi \in \widehat{\Omega}_{c}, \exists p>0\right.$ s.t. there exists $\left(\varphi_{\varepsilon}\right)$ representative of $\varphi$ and $\left(\xi_{\varepsilon}\right)$ representative of $\xi$ such that for any fixed $\left.\varepsilon, \varphi_{\varepsilon}\left(\xi_{\varepsilon}\right)=1, \operatorname{supp}\left(\varphi_{\varepsilon}\right) \subset B\left(\xi_{\varepsilon}, \varepsilon^{p}\right)\right\}$.

$\mathcal{J}_{1}$ is a proper ideal. Let us now prove that $\mathcal{J}_{2}$ is also a proper ideal of $\mathcal{G}(\Omega)$. If this was not the case it would be possible to write $1=f+\sum_{k=0}^{n} g_{k} \varphi_{k}$ where $f \in \mathcal{J}_{1}$ and all $\varphi_{k}$ are elements of $\mathcal{A}$. But as $x_{0} \notin \operatorname{supp}(f)$, there exists a neighbourhood $\omega$ of $x_{0}$ 
s.t. $f / \omega=0$, thus inside $\omega$ we would have $1=\sum_{k=0}^{n} g_{k / \omega} \cdot \varphi_{k / \omega}$. But $\sum_{k=0}^{n} g_{k} \varphi_{k / \omega}$ takes non zero values only on a finite union of sets of the form

$$
\left\{x_{i}(\varepsilon) \text { s.t. }\left|x_{i}(\varepsilon)-x_{k}\right| \leq \varepsilon^{p}, p>0\right\}
$$

the number of these sets and the $p$ being independent of $\varepsilon$. Since for $\varepsilon$ small enough, the measure of this union is less than the measure of $\omega$, this sum cannot take value 1 on all points of $\widehat{\omega}_{c}$.

Consider now $\mathcal{M}$ a maximal ideal containing $\mathcal{J}_{2}$. We will prove that $\mathcal{M}$ cannot be dense. If it was dense its support would be void, thus there would exist a neighbourhood $v$ of $x_{0}$ and an element $\psi$ of $\mathcal{M}$ s.t. $\psi / v=1$. Let now $v^{\prime} \subset \subset v$ be a smaller neighbourhood of $x_{0}$. One can construct a partition of unity $\left(\varphi_{1}, \varphi_{2}\right)$ on $\Omega$ adjusted to the covering $\left(v,{ }^{c} \overline{v^{\prime}}\right)$ i.e. $\operatorname{supp} \varphi_{1} \subset v, \operatorname{supp} \varphi_{2} \subset{ }^{c} \overline{v^{\prime}}$ (where ${ }^{c}$ denotes the complement) and $\varphi_{1}+\varphi_{2}=1$. But by definition $\varphi_{2}$ belongs to $\mathcal{J}_{1}$ and hence to $\mathcal{M}$, hence $1=$ $\varphi_{1}+\varphi_{2}=\varphi_{1} \psi+\varphi_{2} \in \mathcal{M}$ which is impossible since $\mathcal{M}$ is a proper ideal.

Concerning maximal ideals of $\mathcal{G}(\Omega)$ we also have the following property.

Proposition 2.9 If $\mathcal{M}$ is a maximal ideal and is stable under locally finite sums, then $\mathcal{M}$ is closed.

Proof If $\mathcal{M}$ was not closed it would be dense hence $\mathcal{M}$ would contain the ideal $\mathcal{G}_{c}(\Omega)$ (see Corollary 2.3). But if $\left\{\omega_{n}, n \in \mathbb{N}\right\}$ is a countable locally finite covering of $\Omega$ where $\omega_{n}$ are relatively compact open subsets of $\Omega$ and $\varphi_{n}$ is a $C^{\infty}$ partition of unity in $\Omega$ adapted to this covering then all $\left(\varphi_{n}\right)$ would belong to $\mathcal{G}_{c}(\Omega)$ and hence to $\mathcal{M}$ and 1 would also belong to $\mathcal{M}$ since $\mathcal{M}$ is supposed to be stable under locally finite sums.

Proposition 2.10 If $\mathcal{M}$ is a maximal closed ideal of $\mathcal{G}(\Omega)$ then $\mathcal{M} \cap \overline{\mathbb{K}}=m$ is a maximal ideal of $\overline{\mathbb{K}}$.

Proof $\mathcal{M} \cap \overline{\mathbb{K}}$ is a prime ideal of $\overline{\mathbb{K}}$. But $\overline{\mathbb{K}}$ is a topological subspace of $\mathcal{G}(\Omega)$ (here we identify elements of $\overline{\mathbb{K}}$ with the generalized constant functions they define). Thus $\mathcal{M} \cap \overline{\mathbb{K}}$ is a closed prime ideal of $\overline{\mathbb{K}}$, hence a maximal ideal (see [2]).

A natural question is the following: is it always true that if $\mathcal{M}$ is a maximal ideal (dense or closed) then $\mathcal{M} \cap \overline{\mathbb{K}}$ is a maximal ideal of $\overline{\mathbb{K}}$ ?

Unfortunately this is not true as we will see by the following counter example.

Proposition 2.11 There exist dense maximal ideals $\mathcal{M}$ of $\mathcal{G}(\Omega)$ such that $\mathcal{M} \cap \overline{\mathbb{K}}$ is not a maximal ideal of $\overline{\mathbb{K}}$.

Proof Our counterexample will be given in $\mathcal{G}\left(\mathbb{R}^{d}\right)$, the passage to a counterexample in $\mathcal{G}(\Omega)$ for a general $\Omega$ is straightforward but cumbersome. Also for the convenience of the reader, the example will be presented in the sequence model of the theory. (In order to translate the example into the net model, wherever we refer to a representative $\left(f_{n}\right)_{n}$, consider the moderate net $\tilde{f}_{\varepsilon}$ defined by $\left.\left.\left.\forall \varepsilon \in\right] \frac{1}{n+1}, \frac{1}{n}\right], \tilde{f}_{\varepsilon}=f_{n}\right)$. 
Consider now a disjoint family $\mathcal{A}_{n}$ of subsets of $\mathbb{N}$ such that each $\mathcal{A}_{n}$ is infinite and $\bigcup \mathcal{A}_{n}=\mathbb{N}$.

Let $\left(u_{n}\right)$ be a sequence of open subsets of $\mathbb{R}^{d}$ such that $u_{n} \subset B(0, n+1)-B(0, n)$ and for each $u_{i}$ consider a relatively compact open subset $u_{i}^{\prime}$ of $u_{i}$. Let $\left(\varphi_{n}\right)$ be a countable family of $C^{\infty}$ functions such that $0 \leq \varphi_{n} \leq 1 \quad \varphi_{n} / \overline{u_{n}^{\prime}}=1$ and $\operatorname{supp}\left(\varphi_{n}\right)$ is a compact subset of $u_{n}$.

We also choose $u_{n}, \quad u_{n}^{\prime}$ and $\varphi_{n}$ in such a way that all derivatives of all $\varphi_{n}$ are bounded by constants independent of $n$.

Consider now the sequence $\left(g_{n}\right)$ defined by $g_{n}:=\varphi_{k}$ when $n \in \mathcal{A}_{k}$.

Clearly as all $\mathcal{A}_{k}$ are infinite the sequence is not negligible and as all derivatives are bounded the sequence is moderate, so $g=\left[\left(g_{n}\right)\right] \in \mathcal{G}\left(\mathbb{R}^{d}\right)$.

Consider now the following generalized constants (considered as generalized functions on $\mathbb{R}^{d}$ ).

$$
X_{k}=\left[\left(X_{k, n}\right)_{n}\right] \text { where } X_{k, n}=1 \text { if } n \in \mathcal{A}_{k}
$$

(i.e. $X_{k}$ is the class of the characteristic function of the subset $\mathcal{A}_{k}$ ).

Let $I$ be the ideal of $\mathcal{G}\left(\mathbb{R}^{d}\right)$ generated by $(1-g)$ and the family $X_{k}$.

Lemma 2.121 does not belong to $I$.

Proof Suppose the contrary and let $1=\sum_{k=1}^{N} \psi_{k} X_{k}+h(1-g)$, for some $\psi_{k}$ and $h$ in $\mathcal{G}\left(\mathbb{R}^{d}\right)$. Now $\left(\sum_{k=0}^{N} \psi_{k} X_{k}+h(1-g)\right)(x) \neq 1$ for all $x \in \overline{u_{N+1}^{\prime}}$ because this is represented by

$$
z_{n}=\left(\sum_{k=0}^{N} \psi_{k, n} X_{k, n}+h_{n}\left(1-g_{n}\right)\right)(x)
$$

and when $n \in \mathcal{A}_{N+1}$ all $X_{k, n}$ in the above expression are zero and $g_{n}=\varphi_{N+1}$ which implies that $\left(1-g_{n}\right)(x)=\left(1-\varphi_{N+1}\right)(x)$ with $x \in \overline{u_{N+1}^{\prime}}$, thus $\left(1-g_{n}\right)(x)=0$. As $\mathcal{A}_{N+1}$ is infinite, the above sequence $z_{n}$ cannot be equivalent to 1 in the quotient space $\overline{\mathbb{K}}$. Thus 1 does not belong to $I$.

Lemma 2.13 The closure of I contains 1 .

Proof Consider

$$
\Delta=\left[\Delta_{n}\right]=\sum_{k=0}^{\infty}\left[\left(\frac{1}{n}\right)^{k} X_{k, n}\right] \text { and } \theta=\left[\theta_{n}\right]=\sum_{k=0}^{\infty}\left[n^{k} \varphi_{k} X_{k, n}\right]
$$

Both make sense because the sum in the expression of $\Delta_{n}$ converges in $\overline{\mathbb{K}}$ (and thus in $\mathcal{G}\left(\mathbb{R}^{d}\right)$ and for the expression of $\left[\theta_{n}\right]$ the sum is locally finite).

Now we have

$$
\left[\Delta_{n}\right]\left[\theta_{n}\right]=\left[\left(\Delta_{n} \theta_{n}\right)\right]=\sum_{k=0}^{\infty} \varphi_{k} X_{k}=g
$$


(because $X_{k, n} \cdot X_{k, n}=X_{k, n}$ ). of $I$.

$\Delta$ is the limit of $\left[\sum_{k=0}^{N}\left(\frac{1}{n}\right)^{k} X_{k, n}\right]$ which belongs to $I$. Thus $\Delta$ belongs to the closure

Thus $g$ also belongs to the closure $\bar{I}$ of $I$ because $\bar{I}$ is an ideal. But by definition $(1-g)$ belongs to $I$ and hence to $\bar{I}$; thus $1=g+(1-g)$ belongs to $\bar{I}$.

Let now $\mathcal{M}$ be a proper maximal ideal containing $I$. Note now that $\Delta$ does not belong to $\mathcal{M}$. Otherwise, 1 would also belong to $\mathcal{M}$ and $\mathcal{M}$ would not be proper ideal but $\mathcal{M}$ does contain all the generalized constants

$$
A_{N}=\left[\sum_{k=0}^{N}\left(\frac{1}{n}\right)^{k} X_{k, n}\right]
$$

(they belong also to $I \subseteq \mathcal{M}$ ), but the sequence $\left(A_{N}\right)_{N}$ converges to $\Delta$ thus $\mathcal{M} \cap \overline{\mathbb{K}}$ is not closed. This implies that it is not maximal, because all maximal ideals of $\overline{\mathbb{K}}$ are closed.

A natural question is now the following: do there exist dense maximal ideals of $\mathcal{G}(\Omega)$ whose constants are a maximal ideal?

The answer is yes, as can be seen by the following example.

Let $I$ be the ideal spanned in $\mathcal{G}(\Omega)$ by a given maximal ideal $m$ of $\overline{\mathbb{K}}$ and $\mathcal{G}_{c}(\Omega)$. Clearly $I$ is a proper ideal and any maximal ideal $\mathcal{M}$ of $\mathcal{G}(\Omega)$ containing $I$ would verify $\mathcal{M} \cap \overline{\mathbb{K}}=m$.

This remark justifies the following definition.

Definition 2.14 A maximal ideal $\mathcal{M}$ of $\mathcal{G}(\Omega)$ is called a regular maximal ideal if $\mathcal{M} \cap \overline{\mathbb{K}}$ is a maximal ideal of $\overline{\mathbb{K}}$.

In a heuristic sense the problems in the structure of a non regular maximal ideal are concentrated near the border of $\Omega$ and such maximal ideals are not relevant for most natural problems in Analysis.

Proposition 2.15 Let $m$ be a maximal ideal of $\overline{\mathbb{K}}$. Then $\mathcal{J}_{m}(\Omega)$ is the ideal of $\mathcal{G}(\Omega)$ spanned by $m$ (the elements of $m$ being considered as constant generalized functions).

Proof Let $\left(\varphi_{n}\right)$ be a partition of unity of $\Omega$, where $\varphi_{n}$ are elements of $\mathcal{D}(\Omega)$ for which the supports are a locally finite covering of $\Omega$. Let $f$ be an element of $\mathcal{J}_{m}(\Omega)$, $f=\sum \varphi_{n} f$. Let $h_{n, k}$ be the derivatives of all orders (including 0 ) of $\varphi_{n} f$. As $\varphi_{n} f$ has compact support and $f$ belongs to $\mathcal{J}_{m}(\Omega), \alpha_{n, k}=\sup \left|h_{n, k}\right|$ belongs to $m$. For any $n, k \in \mathbb{N}$, consider $q_{n, k}$ such that $\alpha_{n, k}^{\prime}=\left[\left(\varepsilon^{q_{n, k}}\right)\right] \alpha_{n, k}<\left[\left(\varepsilon^{n+k}\right)\right]$. As the valuation of $\alpha_{n, k}^{\prime}$ tends to infinity when $n+k$ tends to infinity, the series $\sum \alpha_{n, k}^{\prime}$ converges to an element $\alpha$ of $m$ (the maximal ideal $m$ being closed). It is clear that there exists $g_{n} \in \mathcal{G}_{c}(\Omega)$ such that $\varphi_{n} f=\alpha g_{n}$ with support of $g_{n}$ included in support of $\varphi_{n}$. Thus $f=\sum \varphi_{n} f=\sum \alpha g_{n}=\alpha \sum g_{n} \in m \mathcal{G}(\Omega)$.

Corollary 2.16 Let $\mathcal{M}$ be a maximal ideal of $\mathcal{G}(\Omega)$ and $m$ be a maximal ideal of $\overline{\mathbb{K}}$. Then the conditions $\mathcal{J}_{m}(\Omega) \subset \mathcal{M}$ and $\mathcal{M} \cap \overline{\mathbb{K}}=m$ are equivalent. 
Our next step will be the characterisation of the family of all regular maximal ideals of $\mathcal{G}(\Omega)$.

Let us now notice that given a maximal ideal $m$ of $\overline{\mathbb{K}}$, if $\pi_{m}$ is the canonical map of $\mathcal{G}(\Omega)$ into $\mathcal{G}_{m}(\Omega)$, then $\pi_{m}$ is continuous and surjective.

Thus if $\mathcal{M}$ is a maximal ideal of $\mathcal{G}(\Omega)$ s.t. $\mathcal{M} \cap \overline{\mathbb{K}}=m$, then $\pi_{m}(\mathcal{M})$ is a maximal ideal of $\mathcal{G}_{m}(\Omega)$ (since $\mathcal{M}$ contains the kernel $\mathcal{J}_{m}(\Omega)$ of $\pi_{m}$ ).

Likewise if $\tilde{\mathcal{M}}$ is a maximal ideal of $\mathcal{G}_{m}(\Omega)$ then $\pi_{m}^{-1}(\tilde{\mathcal{M}})$ is a maximal ideal of $\mathcal{G}(\Omega)$ such that $\pi_{m}^{-1}(\tilde{\mathcal{M}}) \cap \overline{\mathbb{K}}=m$.

Our main result in this paper will be to prove that the family $\Gamma_{r}$ of all regular ideals of $\mathcal{G}(\Omega)$ is in a one to one correspondence with the set of couples $(m, \widehat{\xi})$ where $m$ is a maximal ideal of $\overline{\mathbb{K}}$ and $\widehat{\xi}$ an element of a special compactification of $\widehat{\Omega}_{m, c}$ called the $g$-compactification of $\widehat{\Omega}_{m, c}$ and will be noted $\gamma\left(\widehat{\Omega}_{m, c}\right)$. This compactification is 'smaller' than the Čech compactification of $\widehat{\Omega}_{m, c}$.

We are thus led to the study of ideals and maximal ideals of $\mathcal{G}_{m}(\Omega)$.

\section{Ideals of $\mathcal{G}_{m}(\Omega)$}

\subsection{Generalities}

In the case of $\mathcal{G}_{m}(\Omega)$ the study of ideals is greatly simplified by the fact that the ring of generalized constants is a field and thus $\{0\}$ is the only proper ideal of the ring $\overline{\mathbb{K}}_{m}$.

As $\mathcal{G}_{m}(\Omega)$ has both sheaf properties and continuity properties as a subset of continuous maps from $\widehat{\Omega}_{m, c}$ into $\overline{\mathbb{K}}_{m}$ we have notions of 'support' and 'generalized trace':

Definition 3.1 If $\mathcal{J}$ is an ideal of $\mathcal{G}_{m}(\Omega)$ and $x_{0} \in \Omega$ we say that $x_{0}$ is regular for $\mathcal{J}$ if there exists an open neighbourhood $\mathcal{U}$ of $x_{0}$ s.t. $\mathcal{J} / \mathcal{U}$ contains 1 or equivalently s.t. $\mathcal{J} / \mathcal{U}$ spans $\mathcal{G}_{m}(\mathcal{U})$

Definition 3.2 An $m$-reduced generalized point $\xi$ in $\widehat{\Omega}_{m, c}$ is said to belong to the generalized trace of an ideal $\mathcal{J}$ if $\mathcal{J}(\xi)=0$ (i.e. $\forall f \in \mathcal{J}, f(\xi)=0$ ).

Repeating the proof of Proposition 2.2, one can easily prove:

Proposition 3.3 If $\mathcal{J}$ is a prime ideal then $\operatorname{card}(\operatorname{supp}(\mathcal{J})) \leq 1$.

Proposition 3.4 An ideal $\mathcal{J}$ of $\mathcal{G}_{m}(\Omega)$ is dense if and only if it contains $\mathcal{G}_{m, c}(\Omega)$, i.e. the ideal of compactly supported $m$-reduced generalized functions is the smallest dense ideal of $\mathcal{G}_{m}(\Omega)$. This implies that an ideal is dense if and only if its support is void.

Corollary 3.5 A maximal ideal $\mathcal{M}$ of $\mathcal{G}_{m}(\Omega)$ is closed if and only if

$$
\operatorname{card}(\operatorname{supp}(\mathcal{M}))=1
$$

Concerning generalized trace we can have some more precise properties than in the case of $\mathcal{G}(\Omega)$. 
Proposition 3.6 If a is a prime ideal of $\mathcal{G}_{m}(\Omega)$, then the generalized trace of a has at most one element.

Proof Let us suppose that $\xi_{1}$ and $\xi_{2}$ are two distinct $m$-reduced generalized points. This implies that there exist $p>0$ and nets $\left(\xi_{1, \varepsilon}\right),\left(\xi_{2, \varepsilon}\right)$ representing respectively $\xi_{1}$ and $\xi_{2}$ and their distance net not belonging to $m$, such that for $\varepsilon$ small enough $\left\|\xi_{1, \varepsilon}-\xi_{2, \varepsilon}\right\|>\varepsilon^{p}$. But in this case it is possible to construct two nets $\varphi_{1, \varepsilon}$ and $\varphi_{2, \varepsilon}$, both belonging to $\mathcal{E}_{M}(\Omega)$ such that $\operatorname{supp}\left(\varphi_{1, \varepsilon}\right) \cap \operatorname{supp}\left(\varphi_{2, \varepsilon}\right)=\varnothing$ for $\varepsilon$ small enough and $\varphi_{i, \varepsilon}\left(\xi_{i, \varepsilon}\right)=1$. Let $\varphi_{1}=\left[\left(\varphi_{1, \varepsilon}\right)\right]_{m}, \varphi_{2}=\left[\left(\varphi_{2, \varepsilon}\right)\right]_{m}$. We clearly have $\varphi_{1} \cdot \varphi_{2}=0 \in a$, thus $\varphi_{1}$ or $\varphi_{2}$ has to belong to $a$. But $\varphi_{i}\left(\xi_{i}\right)=1$ thus $\xi_{1}$ or $\xi_{2}$ does not belong to the generalized trace of $a$.

Proposition 3.7 If $\mathcal{M}$ is a maximal ideal and $\{\xi\}$ is the trace of $\mathcal{M}$, then the shadow of $\xi$ is the support of $\mathcal{M}$.

Proof Let $x_{0}$ be the shadow of $\xi$. As $\{\xi\}$ is the generalized trace of $\mathcal{M}$, for any $f \in \mathcal{M}, f(\xi)=0$. If $\left\{x_{0}\right\}$ was not the support of $\mathcal{M}$, there would exist $f \in \mathcal{M}$ and a neighbourhood $V$ of $x_{0}$ such that $f / V=1$ but as $\left|\xi-x_{0}\right|<\frac{1}{n}, \forall n \in \mathbb{N}$, the $m$-reduced generalized point $\xi$ is compactly supported in $V$ thus $f(\xi)=1$ which is impossible.

The existence of maximal closed ideals of $\mathcal{G}_{m}(\Omega)$ with void trace is proved exactly as in the case of $\mathcal{G}(\Omega)$ (the same construction gives a counterexample).

In order to characterize maximal ideals, we will now exhibit important relations between ideals and their 'zero sets'. This will follow the ideas used in [16] when they study ideals of the algebra of real valued continuous functions on a completely regular Hausdorff space.

\subsection{Generalized zero sets of $\widehat{\boldsymbol{\Omega}}_{m, c}$}

Definition 3.8 A subset $A$ of $\widehat{\Omega}_{m, c}$ is a generalized zero set $\left(Z_{g}\right)$ if there exists $f \in$ $\mathcal{G}_{m}(\Omega)$ s.t. $A=\left\{\xi \in \widehat{\Omega}_{m, c}\right.$ s.t. $\left.f(\xi)=0\right\}$. In this case we note $A=Z_{g}(f)$. More generally if $\mathcal{B}$ is any subset of $\mathcal{G}_{m}(\Omega), Z_{g}(\mathcal{B})$ will be the family of all $Z_{g}(f)$ for $f \in \mathcal{B}$.

Clearly, as $m$-reduced generalized functions define continuous mappings from $\widehat{\Omega}_{m, c}$ into $\overline{\mathbb{K}}$, it is clear that all generalized zero sets are closed subsets of $\widehat{\Omega}_{m, c}$.

A natural question is the following: is any given closed subset of $\widehat{\Omega}_{m, c}$ a generalized zero set? The answer is no.

Proposition 3.9 There exist closed subsets of $\widehat{\Omega}_{m, c}$ which are $\operatorname{not} Z_{g}-$ sets.

The proof is based on the following lemma.

Lemma 3.10 If $\Omega$ is connected and $A$ and $B$ are two complementary subsets of $\widehat{\Omega}_{m, c}$, they cannot both be $Z_{g}$-sets. 
Proof Let $f$ be such that $Z_{g}(f)=A$ and $g$ such that $Z_{g}(g)=B$. Clearly $Z_{g}(f)=$ $Z_{g}\left(|f|^{2}\right)$ and $Z_{g}(g)=Z_{g}\left(|g|^{2}\right)$. Notice that

$$
Z_{g}\left(|f|^{2}+|g|^{2}\right)=Z_{g}\left(|f|^{2}\right) \cap Z_{g}\left(|g|^{2}\right)=\emptyset .
$$

Thus $\forall \xi \in \widehat{\Omega}_{m, c},\left(|g|^{2}+|f|^{2}\right)(\xi) \neq 0$.

This implies that $|g|^{2}+|f|^{2}$ is invertible (Proposition 1.24). Consider now the reduced generalized function

$$
h=\frac{|f|^{2}}{|f|^{2}+|g|^{2}}
$$

Clearly, $\forall \xi \in A h(\xi)=0$ and $\forall \xi \in B h(\xi)=1$. As $A$ and $B$ are complementary, $h$ takes only the values 0 and 1 . This is contradictory to the intermediate value property for $m$-reduced generalized functions on a connected open set.

Now notice that in $\widehat{\Omega}_{m, c}$ there exist couples of complementary closed subsets, for example:

let $A$ be the halo of zero and $B$ be the complement of $A$. Then one can easily verify that $A$ and $B$ are both closed.

Proposition 3.11 The family of $Z_{g}$-sets is stable under countable intersection.

Proof For each $Z_{g}$-set $Z_{g}(f)$, notice that $Z_{g}(f)=Z_{g}\left(\left[\left(\varepsilon^{n}\right)\right]|f|^{2}\right)$.

Let $\left(Z_{g}\left(f_{n}\right)\right)$ be a sequence of $Z_{g}$-sets. Let $\left(\mu_{n}\right)$ be an increasing sequence of seminorms defining the topology of $C^{\infty}(\Omega)$. Then we can find positive integers $m_{n}$ such that $\mu_{n}\left(\left[\left(\varepsilon^{m_{n}}\right)\right]\left|f_{n}\right|^{2}\right) \leq\left[\left(\varepsilon^{n}\right)\right], \forall n$. Then

$$
\bigcap Z_{g}\left(f_{n}\right)=Z_{g}\left(\sum\left[\left(\varepsilon^{m_{n}}\right)\right]\left|f_{n}\right|^{2}\right) .
$$

Hence a countable intersection of generalized zero sets is a generalized zero set, too.

Proposition 3.12 The family of $Z_{g}$-sets is a base for the family of closed subsets of $\widehat{\Omega}_{m, c}$ i.e. every closed subset is an intersection of $Z_{g}-$ sets.

This is an easy consequence of the following lemma:

Lemma 3.13 If $A$ is a closed subset of $\widehat{\Omega}_{m, c}$ and $\xi \notin A$, then there exists $f \in \mathcal{G}_{m}(\Omega)$ s.t. $f(\xi)=1$ and $\forall r \in A \quad f(r)=0$ (i.e., $Z_{g}(f) \supset A$ ).

Proof $A$ being closed, there exists a positive constant $p$ such that $B\left(\xi,\left[\left(\varepsilon^{p}\right)\right]\right) \cap A=\emptyset$. Consider now a net $\left(\xi_{\varepsilon}\right)$ representing $\xi$. Let $\varphi \in \mathcal{D}\left(\mathbb{R}^{n}\right)$ s.t. $0 \leq \varphi \leq 1, \varphi(0)=1$ and $\operatorname{supp}(\varphi) \subset B(0,1)$. Consider the net $\varphi_{\varepsilon}(x)=\varphi\left(\frac{x-\xi_{\varepsilon}}{\varepsilon^{p}}\right)$. Clearly this net is moderate and for $\varepsilon$ small enough, its support is included in a relatively compact subset of $\Omega$. Thus we can define $\left[\left(\varphi_{\varepsilon}\right)\right]_{m}=f$. One can now easily verify that $f$ satisfies the requirements of the lemma. 


\section{3 $Z_{g}$-sets, $Z_{g}-$ filters}

As we already saw, a $Z_{g}$-set is a subset of $\widehat{\Omega}_{m, c}$ s.t. there exists an $m$ - reduced generalized function $f$ s.t. $f$ takes zero values on all elements of this subset and only there.

These sets will play a role analogous to $z-$ sets in [16] for the investigation of properties of ideals and the study of the family of maximal ideals.

Definition 3.14 A $Z_{g}$-filter $\mathcal{F}$ is a family of $Z_{g}$-sets such that

(a) if $A$ is a $Z_{g}$-set, $A \supset B$ and $B \in \mathcal{F}$, then $A \in \mathcal{F}$.

(b) if $\left(A_{n}\right)_{n \leq N}$ is a finite family of elements of $Z_{g}$, then $\bigcap_{n \leq N} A_{n} \in \mathcal{F}$.

(c) $\emptyset$ does not belong to $\mathcal{F}$.

Proposition 3.15 Given a $Z_{g}$-filter $\mathcal{F}$, the family $\mathcal{J}(\mathcal{F})$ of all $m$-reduced generalized functions on $\Omega$ whose generalized zero set belong to $\mathcal{F}$ is a proper ideal.

Proof If $f \in \mathcal{G}_{m}(\Omega)$ and $g \in \mathcal{J}(\mathcal{F})$, then $Z_{g}(f g) \supset Z_{g}(g) \in \mathcal{F}$. Thus $Z_{g}(f g) \in \mathcal{F}$ and $f g \in \mathcal{J}(\mathcal{F})$.

If $f \in \mathcal{J}(\mathcal{F})$ and $g \in \mathcal{J}(\mathcal{F})$, then

$$
Z_{g}(f+g) \supset Z_{g}(f) \cap Z_{g}(g) \in \mathcal{F} \text {. }
$$

Thus $f+g \in \mathcal{J}(f)$.

This leads to the following definition.

Definition 3.16 An ideal $\mathcal{J}$ is called a $Z_{g}$-ideal if it is of the form $\mathcal{J}(\mathcal{F})$ for some $Z_{g}$ - filter $\mathcal{F}$.

Example Let $A$ be a nonempty subset of $\widehat{\Omega}_{m, c}$ and consider the ideal $\mathcal{J}$ of all elements of $\mathcal{G}_{m}(\Omega)$ which take the value zero on all elements of $A$. Then $\mathcal{J}=\mathcal{J}(\mathcal{F})$, where $\mathcal{F}$ is the family of all $Z_{g}$-sets which contain $A$. One can easily verify that $\mathcal{F}$ is a $Z_{g}$ - filter.

There exist ideals which are not of this type: consider the ideal $\mathcal{J}_{1}$ of $\mathcal{G}_{m}(\mathbb{R})$ spanned by $x$ and consider the ideal $\mathcal{J}_{2}$ spanned by $x^{2}$. Those ideals have the same family of $Z_{g}$-sets, but they are not the same.

Definition 3.17 Given an ideal $\mathcal{J}$, we denote $\mathcal{F}(\mathcal{J})$ the family of all $Z_{g}$-sets of elements of $\mathcal{J}$.

Proposition 3.18 If $\mathcal{J}$ is a proper ideal of $\mathcal{G}_{m}(\Omega)$, then $\mathcal{F}(\mathcal{J})$ is a $Z_{g}$-filter.

Proof Let $A=Z_{g}(f)$, and $B=Z_{g}(g)$ where $g \in \mathcal{J}$. Let us suppose that $A \supset B$. In this case $Z_{g}(f)=Z_{g}(f g)$, because $f g(\xi)=f(\xi) g(\xi)=0$ if and only if $f(\xi)$ is zero or $g(\xi)$ is zero ( $\overline{\mathbb{K}}$ is a field). But as $Z_{g}(f) \supset Z_{g}(g)$, we see that $f(x)$ is zero if and only if $f g(x)$ is zero. But as $f g \in \mathcal{J}$, we have proved that $A=Z_{g}(f)=Z_{g}(f g)$ belongs to $\mathcal{F}(\mathcal{J})$. 
Let now $A$ and $B$ belong to $\mathcal{F}(\mathcal{J}) \quad\left(A=Z_{g}(f), B=Z_{g}(g)\right.$ with $\left.(f, g) \in \mathcal{J}^{2}\right)$. Then as $Z_{g}(f)=Z_{g}\left(|f|^{2}\right)$ and $Z_{g}(g)=Z_{g}\left(|g|^{2}\right)$, we have $A \cap B=Z_{g}\left(|f|^{2}+|g|^{2}\right)$. But $|f|^{2}+|g|^{2} \in \mathcal{J}$, thus $A \cap B \in \mathcal{F}(\mathcal{J})$.

Finally, $\emptyset \notin \mathcal{F}(\mathcal{J})$ because of Proposition 1.24.

Proposition 3.19 If $\mathcal{J}$ is a $Z_{g}-$ ideal, and if $f \in \mathcal{G}_{m}(\Omega)$ is such that $|f|^{2} \in \mathcal{J}$, then $f \in \mathcal{J}$.

Proof This is a direct consequence of the fact that $Z_{g}(f)=Z_{g}\left(|f|^{2}\right)$.

Proposition 3.20 If $\mathcal{J}_{1} \subset \mathcal{J}_{2}$ and $\mathcal{J}_{1}$ and $\mathcal{J}_{2}$ are proper ideals of $\mathcal{G}_{m}(\Omega)$, then $\mathcal{F}\left(\mathcal{J}_{1}\right) \subset$ $\mathcal{F}\left(\mathcal{J}_{2}\right)$. If $\mathcal{F}_{1}$ and $\mathcal{F}_{2}$ are two $Z_{g}-$ filters, $\mathcal{F}_{1} \subset \mathcal{F}_{2}$, then $\mathcal{J}\left(\mathcal{F}_{1}\right) \subset \mathcal{J}\left(\mathcal{F}_{2}\right)$. If $\mathcal{P}$ is a $Z_{g}$-ideal, then $\mathcal{P}=\mathcal{J}\left(\mathcal{F}(\mathcal{P})\right.$ ) and if $\mathcal{F}$ is a $Z_{g}$-filter, then $\mathcal{F}(\mathcal{J}(\mathcal{F}))=\mathcal{F}$. If $\mathcal{P}$ is any proper ideal of $\mathcal{G}_{m}(\Omega)$, then $\mathcal{P} \subset \mathcal{J}(\mathcal{F}(\mathcal{P})$ ).

The proof is a direct consequence of the definitions.

By Zorn's theorem, one can easily verify that any $Z_{g}-$ filter is included is some maximal $Z_{g}-$ filter.

Definition 3.21 Maximal $Z_{g}$-filters will be called $Z_{g}$-ultrafilters.

There is a correspondence between maximal ideals and maximal $Z_{g}-$ ultrafilters:

Proposition 3.22 If $\mathcal{M}$ is a maximal ideal of $\mathcal{G}_{m}(\Omega)$, then $\mathcal{F}(\mathcal{M})$ is a $Z_{g}$-ultrafilter and conversely, if $\mathcal{F}$ is a $Z_{g}$-ultrafilter, then $\mathcal{J}(\mathcal{F})$ is a maximal ideal of $\mathcal{G}_{m}(\Omega)$.

Proof If $\mathcal{F}(\mathcal{M})=\mathcal{F}_{1}$ was not maximal there would exist a $Z_{g}$-ultrafilter $\mathcal{F}_{2}$ strictly larger than $\mathcal{F}_{1}$ and thus there would exist $f \in \mathcal{J}\left(\mathcal{F}_{2}\right)$ s.t. $Z_{g}(f) \notin \mathcal{F}(\mathcal{M})$. Hence $\mathcal{J}\left(\mathcal{F}_{2}\right)$ would be a proper ideal strictly larger than $\mathcal{M}$.

Likewise, let us suppose that $\mathcal{J}(\mathcal{F})$ is not maximal. Then there would exist a proper ideal $\mathcal{P}$ strictly larger than $\mathcal{J}(\mathcal{F})$. It would thus have an element $f$ s.t. $Z_{g}(f) \notin \mathcal{F}$; thus $\mathcal{F}(\mathcal{P})$ would be a $Z_{g}-$ filter larger than $\mathcal{F}$. Hence $\mathcal{F}$ would not be an ultrafilter.

It is now clear that maximal ideals are in a one to one correspondence with $Z_{g}$-ultrafilters. This will allow us to consider the set of all $Z_{g}$-ultrafilters and prove later that it gives a compactification of $\widehat{\Omega}_{m, c}$.

But let us first give some more properties of $Z_{g}$-filters and their associated ideals. As in the case of ideals we have a notion of 'support' of $Z_{g}-$ filters.

Definition 3.23 A point $x_{0} \in \Omega$ is said to be regular for a $Z_{g}-$ filter $\mathcal{F}$ if there exists a neighbourhood $V$ of $x_{0}$ s.t. there exist $A \in \mathcal{F}$ s.t. the shadow of every element of $A$ is in the complement of $V$.

The support of a $Z_{g}$-filter $\mathcal{F}$ is the complement of the open set of all regular points for $\mathcal{F}$. It will be denoted $\operatorname{supp}(\mathcal{F})$. There is a natural relation between supports of $Z_{g}-$ filters and supports of associated ideals.

Proposition 3.24 If $\mathcal{F}$ is a $Z_{g}-$ filter, then $\operatorname{supp}(\mathcal{F})=\operatorname{supp}(\mathcal{J}(\mathcal{F})$ ).

Likewise, if $\mathcal{P}$ is an ideal of $\mathcal{G}_{m}(\Omega)$, then $\operatorname{supp}(\mathcal{P})=\operatorname{supp}(\mathcal{F}(\mathcal{P}))$. 
Proof If $x_{0} \notin \operatorname{supp}(\mathcal{F})$, then there exists a ball $B\left(x_{0}, \rho\right) \subset \Omega$ s.t. there exists $A \subset$ $\widehat{\Omega}_{m, c}, A \in \mathcal{F}$ s.t. $B\left(x_{0}, \rho\right)$ does not contain any shadow of an element of $A$. Consider $\varphi \in \mathcal{D}(\Omega)$ s.t. $\operatorname{supp}(\varphi) \subset B\left(x_{0}, \rho\right)$ and $\varphi / B\left(x_{0}, \frac{\rho}{2}\right)=1 . Z_{g}(\varphi)$ is in the complement of $\widehat{B\left(x_{0}, \rho\right)}$ (considered as a part of $\left.\widehat{\Omega}_{m, c}\right)$. Thus $Z_{g}(\varphi) \supset A$, thus $Z_{g}(\varphi) \in \mathcal{F}$, thus $\varphi \in \mathcal{J}(\mathcal{F})$ and $\varphi=1$ on a neighbourhood $V$ of $x_{0}$, hence $x_{0}$ is regular for $\mathcal{J}(\mathcal{F})$. There exists a neighbourhood $V$ of $x_{0}$ and $f_{0} \in \mathcal{J}(\mathcal{F})$ s.t. $f_{0} / V=1$. Thus the zero set of $\mathcal{F}_{0}$ has a shadow that does not intersect $V$. An analogous argument holds for regular points of $\mathcal{P}$ and $\mathcal{F}(\mathcal{P})$.

Definition 3.25 A $Z_{g}$-filter $\mathcal{F}$ is said to be prime if, whenever the union $A \cup B$ of two $Z_{g}$-sets $A$ and $B$ belongs to $\mathcal{F}$, then $A \in \mathcal{F}$ or $B \in \mathcal{F}$.

In the case of $Z_{g}$-ideals, there is a natural relation between the notion of prime ideal and prime $Z_{g}$-filter.

Proposition 3.26 Let $\mathcal{P}=\mathcal{J}(\mathcal{F})$. Then $\mathcal{P}$ is prime if and only if $\mathcal{F}$ is prime.

Proof

$$
f \cdot g \in \mathcal{P} \Longleftrightarrow Z_{g}(f) \cup Z_{g}(g)=Z_{g}(f \cdot g) \in \mathcal{F} \text {. }
$$

Let $Z_{g}(f) \cup Z_{g}(g) \in \mathcal{F}$. If $\mathcal{P}$ is prime, then $Z_{g}(f) \in \mathcal{F}$ or $Z_{g}(g) \in \mathcal{F}$, thus $\mathcal{F}$ is prime. The converse argument is of the same kind.

\subsection{The compact set $\gamma\left(\widehat{\Omega}_{m, c}\right)$}

Following the ideas of the Čech-compactification, we will now see how the family of $Z_{g}$-ultrafilters constitutes a compact space into which $\widehat{\Omega}_{m, c}$ is densely embedded. The main idea will be to show that the family of $Z_{g}$-sets is a compactifying family for $\widehat{\Omega}_{m, c}$ (see Appendix).

Proposition 3.27 The family $Z_{g}$ of $Z_{g}$-sets satisfies the following properties:

(1) $\widehat{\Omega}_{m, c} \in Z_{g}$.

(2) For any closed subset $F$ of $\widehat{\Omega}_{m, c}$ and $x \in{ }^{c} F$, there exists $F^{\prime} \in Z_{g}$ s.t. $x \notin F^{\prime}$ and $F^{\prime} \supset F$.

(3) For any couple $F_{1}, F_{2}$ of elements of $Z_{g}$ s.t. $F_{1} \cap F_{2}=\emptyset$, there exist $F_{1}^{\prime}, F_{2}^{\prime}$ elements of $Z_{g}$ s.t.

$$
F_{1} \subset F_{1}^{\prime}, F_{2} \subset F_{2}^{\prime}, F_{1}^{\prime} \cap F_{2}=F_{2}^{\prime} \cap F_{1}=\emptyset, F_{1}^{\prime} \cup F_{2}^{\prime}=\widehat{\Omega}_{m, c} .
$$

(4) $Z_{g}$ is stable under countable intersections and under finite unions.

5) If $x \in \widehat{\Omega}_{m, c}$, then $\{x\} \in Z_{g}$.

Proof (1) $\widehat{\Omega}_{m, c}=Z_{g}(0)$.

(2) It is exactly the content of Lemma 3.13. 
(3) Let us suppose $F_{1}=Z_{g}(|f|)=Z_{g}\left(|f|^{2}\right), \quad F_{2}=Z_{g}(g)=Z_{g}\left(|g|^{2}\right)$. Consider now the functions $\tilde{f}=\frac{|f|^{2}}{|f|^{2}+|g|^{2}}$ and $\tilde{g}=\frac{|g|^{2}}{|f|^{2}+|g|^{2}}$. Clearly, $Z_{g}(\tilde{f})=F_{1}$ and $Z_{g}(\tilde{g})=F_{2}$. Moreover

$$
F_{2}=\left\{\xi \in \widehat{\Omega}_{m, c} \text { s.t. } \tilde{f}(\xi)=1\right\}
$$

We will use the following lemma:

Lemma 3.28 Let $f \in \mathcal{G}_{m}(\Omega)$ and let a be a positive real number. Then there exists $g \in \mathcal{G}_{m}(\Omega)$ s.t. $\left\{x,|f(x)|^{2} \leq a\right\} \subset Z_{g}(g) \subset\left\{x,|f(x)|^{2} \leq a+\eta\right\}$ for each real number $\eta>0$.

Proof Let $\phi$ be a $C^{\infty}$ increasing function on $\mathbb{R}$ s.t. $\phi(x)=0$ iff $x \leq a$, and s.t. for $x \geq 2 a, \phi(x)=1$. Clearly $\phi$ is a bounded smooth function and $\phi^{\prime}$ has compact support, thus $g=\phi\left(|f|^{2}\right) \in \mathcal{G}_{m}(\Omega)$. Clearly $g$ takes value zero on all compactly supported generalized points $x$ such that $|f(x)|^{2} \leq a$. Thus $Z_{g}(g) \supset\left\{x,|f(x)|^{2} \leq a\right\}$. Likewise, if $|f(x)|^{2}>a+\eta$, then clearly $\phi\left(|f|^{2}\right)>0$, thus

$$
\left\{x,|f(x)|^{2} \leq a+\eta\right\} \supset Z_{g}(g) \supset\left\{x,|f(x)|^{2} \leq a\right\}
$$

Consider now $h_{1} \in \mathcal{G}_{m}(\Omega)$ s.t. for any real $\eta>0$

$$
\left\{x, \tilde{f}(x) \leq \frac{1}{2}\right\} \subset Z_{g}\left(h_{1}\right) \subset\left\{x, \tilde{f}(x) \leq \frac{1}{2}+\eta\right\}
$$

and $h_{2} \in \mathcal{G}_{m}(\Omega)$ s.t.

$$
\left\{x, \tilde{f}(x) \geq \frac{1}{2}-\eta\right\} \supset Z_{g}\left(h_{2}\right) \supset\left\{x, \tilde{f}(x) \geq \frac{1}{2}\right\} .
$$

The existence of $h_{1}$ and $h_{2}$ can easily be deduced by the previous lemma. Put now $F_{1}^{\prime}=Z_{g}\left(h_{1}\right)$ and $F_{2}^{\prime}=Z_{g}\left(h_{2}\right)$. Clearly,

$$
F_{1}^{\prime} \cup F_{2}^{\prime}=\widehat{\Omega}_{m, c}, \quad F_{1}^{\prime} \supset F_{1}, \quad F_{2}^{\prime} \supset F_{2}, \quad F_{1} \cap F_{2}^{\prime}=\emptyset, \quad F_{2} \cap F_{1}^{\prime}=\emptyset .
$$

(4) By Proposition 3.11 and because $Z_{g}(f) \cup Z_{g}(g)=Z_{g}(f g)$.

(5) Let $\left(x_{\varepsilon}\right)$ be a net representing $x$ and consider $f_{\varepsilon}(t)=\left(t-x_{\varepsilon}\right) / \Omega$. Clearly, $Z_{g}\left[\left(f_{\varepsilon}\right)\right]_{m}=\left\{\left[\left(x_{\varepsilon}\right)\right]_{m}\right\}=\{x\}$.

Now we can conclude using Theorem A.2 of Appendix that $Z_{g}$-sets constitute a compactifying family and thus that the family $\gamma\left(\widehat{\Omega}_{m, c}\right)$ of $Z_{g}-$ ultrafilters constitutes a compactification of $\widehat{\Omega}_{m, c}$. (Recall that $\widehat{\Omega}_{m, c}$ is metrizable, hence normal.)

A natural question now is if $\gamma\left(\widehat{\Omega}_{m, c}\right)$ is the Čech-compactification of $\widehat{\Omega}_{m, c}$ (denoted $\left.\beta\left(\widehat{\Omega}_{m, c}\right)\right)$. 
This is not the case. To prove it, we will use Theorem B.1 of Appendix and thus we only have to prove that the there exists a pair $\left(F_{1}, F_{2}\right)$ of disjoint closed subsets of $\widehat{\Omega}_{m, c}$ such that there does not exist a pair $\widehat{F}_{1}, \widehat{F}_{2}$ of $Z_{g}-$ sets s.t. $F_{1} \subset \widehat{F}_{1}, F_{2} \subset \widehat{F}_{2}$ and $\widehat{F}_{1} \cap \widehat{F}_{2}=\emptyset$. This is obvious because we saw that there exist pairs of complementary closed subsets of $\widehat{\Omega}_{m, c}$, but there does not exist a pair of complementary $Z_{g}$-sets.

We have at last proved the main theorem of this section.

Theorem 3.29 The family of maximal ideals of $\mathcal{G}_{m}(\Omega)$ is in a one to one correspondence with $\gamma\left(\widehat{\Omega}_{m, c}\right)$ which is a compactification of $\widehat{\Omega}_{m, c}$ different from the Čech-compactification of $\widehat{\Omega}_{m, c}$.

By pull back we obtain the following:

Theorem 3.30 The family of regular maximal ideals $\mathcal{M}$ of $\mathcal{G}(\Omega)$ is in a one to one correspondence with couples $(m, \xi)$ where $m=\mathcal{M} \cap \overline{\mathbb{K}}$ is a maximal ideal of $\overline{\mathbb{K}}$ and $\xi$ is an element of $\gamma\left(\widehat{\Omega}_{m, c}\right)$.

We have thus classified all regular maximal ideals, but we still have no idea concerning the family of non-regular maximal ideals (i.e., the dense maximal ideals $\mathcal{M}$ s.t. $\mathcal{M} \cap \overline{\mathbb{K}}$ is not a maximal ideal of $\overline{\mathbb{K}}$ ).

There is also another field of analogous investigations. In [5], the authors investigated the behaviour of objects of the theory under the additional hypothesis that we impose parametrizations by $\varepsilon$ to be continuous on $\varepsilon$. Which results of this paper are still valid under this condition? For the case of generalized constants, a first investigation has been done in [18].

Acknowledgements We thank very well the referee for his judicious remarks and Catherine Gourion for the idea of ultrafilter of closed sets for theorem A1 of Appendix.

Open Access This article is licensed under a Creative Commons Attribution 4.0 International License, which permits use, sharing, adaptation, distribution and reproduction in any medium or format, as long as you give appropriate credit to the original author(s) and the source, provide a link to the Creative Commons licence, and indicate if changes were made. The images or other third party material in this article are included in the article's Creative Commons licence, unless indicated otherwise in a credit line to the material. If material is not included in the article's Creative Commons licence and your intended use is not permitted by statutory regulation or exceeds the permitted use, you will need to obtain permission directly from the copyright holder. To view a copy of this licence, visit http://creativecommons.org/licenses/by/4.0/.

\section{A Compactifying families of closed subsets}

Let us first remind the definition of a normal topological space $E$. A Hausdorff topological space $E$ is said to be normal if for any couple $F_{1}, F_{2}$ of disjoint closed subsets there exists a couple $\left(U_{1}, U_{2}\right)$ of disjoint open subsets such that $F_{1} \subset U_{1}, F_{2} \subset U_{2}$.

In this appendix we will show that some families of closed subsets of a normal space $E$ allow us to define a compactification $\tilde{E}$ of $E$.

Definition A.1 A family $\mathcal{S}$ of closed subsets of a normal topological space $E$ is a compactifying family of closed subsets if the following conditions hold:

(1) $E \in \mathcal{S}$. 
(2) For any closed subset $F$ of $E$ and every element $x$ in the complement ${ }^{c} F$ of $F$ there exists $F^{\prime} \in \mathcal{S}$ s.t. $F^{\prime} \supset F$ and $x \in{ }^{c} F^{\prime}$.

(3) For any couple $F_{1}, F_{2}$ of elements of $\mathcal{S}$ s.t. $F_{1} \cap F_{2}=\emptyset$ There exist $F_{1}^{\prime}, F_{2}^{\prime}$ elements of $\mathcal{S}$ s.t.

$$
\begin{array}{r}
F_{1} \subset F_{1}^{\prime}, F_{2} \subset F_{2}^{\prime}, F_{1}^{\prime} \cup F_{2}^{\prime}=E, F_{1} \cap F_{2}^{\prime}=F_{2} \cap F_{1}^{\prime}=\emptyset . \\
\text { (i.e. } \left.F_{1} \subset{ }^{c} F_{2}^{\prime}, F_{2} \subset{ }^{c} F_{1}^{\prime}, \text { and }{ }^{c} F_{1}^{\prime} \cap{ }^{c} F_{2}^{\prime}=\emptyset\right)
\end{array}
$$

(4) $\mathcal{S}$ is stable under finite intersections and under finite unions.

(5) If $x \in E, \quad\{x\} \in \mathcal{S}$.

Theorem A.2 Let $E$ be a normal topological space and $\mathcal{H}$ a compactifying family of closed subsets of $E$.

Let $\tilde{\mathcal{H}}$ be the set of all $\mathcal{H}$-filters (i.e., filters of elements of $\mathcal{H}$ ). Let $\widehat{E}_{\mathcal{H}}$ be the set of $\mathcal{H}$-ultrafilters, i.e. the set of maximal $\mathcal{H}$-filters. Consider now the following family of subsets of $\widehat{E}_{\mathcal{H}}$

$$
\widehat{\mathcal{O}}=\left\{\widehat{E}_{\mathcal{H}}\right\} \cup\left\{\mathcal{O}_{F}: F \in \tilde{\mathcal{H}}\right\}
$$

where

$$
\mathcal{O}_{F}:=\left\{G \in \widehat{E}_{\mathcal{H}}, F \not \subset G\right\} \quad(F \in \tilde{\mathcal{H}}) .
$$

Then (a) $\widehat{\mathcal{O}}$ is a basis of open sets for some topology on $\widehat{E}_{\mathcal{H}}$.

(b) The mapping $u: E \rightarrow \widehat{E}_{\mathcal{H}}$ defined by

$$
u(x)=\{F \in \mathcal{H} \text { s.t. } x \in F\} \text { (this is clearly a } \mathcal{H} \text {-ultrafilter) }
$$

is injective and continuous.

(c) $\widehat{E}_{\mathcal{H}}$ is a compact Hausdorff space and $E$ is embedded by $u$ into a dense subset of $\widehat{E}_{\mathcal{H}}$.

$\widehat{E}_{\mathcal{H}}$ will be called the $\mathcal{H}$-compactification of $E$.

Proof (a) The family $\widehat{\mathcal{O}}$ defines a basis of a topology:

$\emptyset \in \widehat{\mathcal{O}}$, since $\emptyset=\mathcal{O}_{\{E\}}$.

$\widehat{E}_{\mathcal{H}} \in \widehat{\mathcal{O}}$ by definition.

If $\left\{\mathcal{O}_{i}, i \in I\right\}$ is a family of elements of $\widehat{\mathcal{O}}$, then either $\mathcal{O}_{i}=\widehat{E}_{\mathcal{H}}$ for some $i$ (in which case trivially $\bigcup \mathcal{O}_{i} \in \widehat{\mathcal{O}}$ ) or $\mathcal{O}_{i}=\mathcal{O}_{F_{i}}, \forall i$. Then if $\bigcup \mathcal{O}_{i} \neq \widehat{E}_{\mathcal{H}}$, we have $\cup \mathcal{O}_{i}=\mathcal{O}_{F}$, where $F$ is the smallest $\mathcal{H}$-filter containing all $F_{i}$. This is possible because if $\bigcup \mathcal{O}_{i} \neq E_{\mathcal{H}}$, there exists a $\mathcal{H}$-ultrafilter $B$ such that $B$ does not belong to any $\mathcal{O}_{i}$. This means by definition that all $F_{i}$ are included in $B$. Thus there exists a minimal $\mathcal{H}$-filter $F$ containing all $F_{i}$.

This means that $\bigcup \mathcal{O}_{i}=\left\{G \in \widehat{E}_{\mathcal{H}}, F \not \subset G\right\}$, i.e. $\bigcup \mathcal{O}_{i} \in \widehat{\mathcal{O}}$.

As $\mathcal{H}$ is stable under finite unions, and every $\mathcal{H}$-ultrafilter is prime (by the same argument as $[16,2.13])$, we have that $\mathcal{O}_{F_{1}} \cap \mathcal{O}_{F_{2}}=\mathcal{O}_{F_{1} \cap F_{2}}$.

Thus $\widehat{\mathcal{O}}$ satisfies the condition to be the family of open subsets of some topology. 
(b) Clearly, $\{F \in \mathcal{H}, x \in F\}$ is a well defined $\mathcal{H}$-ultrafilter, $E$ being a normal Hausdorff space. Since an $\mathcal{H}$-ultrafilter contains at most one singleton (property of non empty intersection), and since for every $x$ of $E$ the only singleton of $u(x)$ is $\{x\}$, the mapping $u$ is injective. We will now show the continuity of $u$. i.e., if $\mathcal{O} \in \widehat{\mathcal{O}}$, $u^{-1}(\mathcal{O})$ is an open subset of $E$.

$$
\begin{aligned}
& \text { If } \mathcal{O}=\widehat{E}_{\mathcal{H}}, u^{-1}(\mathcal{O})=E \text {. If now } \mathcal{O}=\mathcal{O}_{F} \text {, then } \\
& \qquad u^{-1}(\mathcal{O})=\{x \text { s.t. } F \not \subset u(x)\}=\{x \text { s.t. } \exists D \in F \text { s.t. } x \notin D\}
\end{aligned}
$$

which is clearly an open set.

Let now $W$ be an open subset of $E$ and $D=E-W$. Let $F$ be the $\mathcal{H}-$ filter of all elements of $\mathcal{H}$ containing $D$. It is now easy to verify that $W=u^{-1}\left(\mathcal{O}_{F}\right)$. Thus the trace of our topology on $u(E)$ 'coincides' with the topology on $E$.

(c) To see that $u(E)$ is dense in $\widehat{E}_{\mathcal{H}}$, we only have to show that if $\mathcal{O}_{F}$ is a non void open subset of $\widehat{E}_{\mathcal{H}}$, then $u^{-1}\left(\mathcal{O}_{F}\right)$ is a non void subset of $E$. But this is obvious, since

$$
u^{-1}\left(\mathcal{O}_{F}\right)=\{x \text { s.t. } \exists D \in F \text { s.t. } x \notin D\} \neq \varnothing .
$$

Let us now prove that $\widehat{E}_{\mathcal{H}}$ is Hausdorff. Let $\widehat{x}_{1}$ and $\widehat{x}_{2}$ be two distinct $\mathcal{H}$-ultrafilters. Then there exist $D_{1} \in \widehat{x}_{1}$ and $D_{2} \in \widehat{x}_{2}$ s.t. $D_{1} \cap D_{2}=\emptyset$. (Indeed, if $D_{1} \in \widehat{x}_{1}$ is such that $D_{1} \cap D_{2} \neq \varnothing$, for each $D_{2} \in \widehat{x}_{2}$, then there exists an $\mathcal{H}$-filter generated by $\widehat{x}_{2}$ and $D_{1}$. By maximality, this $\mathcal{H}$-filter coincides with $\widehat{x}_{2}$. Thus $D_{1} \in \widehat{x}_{2}$.) Then by property 3 of Definition A.1, there exist $D_{1}^{\prime}, D_{2}^{\prime}$ elements of $\mathcal{H}$ s.t.

$$
D_{1} \subset D_{1}^{\prime}, D_{2} \subset D_{2}^{\prime}, D_{1} \subset{ }^{c} D_{2}^{\prime}, D_{2} \subset{ }^{c} D_{1}^{\prime}, D_{1}^{\prime} \cup D_{2}^{\prime}=E
$$

Let $F_{i}^{\prime}$ be the $\mathcal{H}$-filter of all elements of $\mathcal{H}$ containing $D_{i}^{\prime}$. Since $D_{1}^{\prime} \cup D_{2}^{\prime}=E$, a maximal $\mathcal{H}$-filter either contains $D_{1}^{\prime}$ or contains $D_{2}^{\prime}$ (or both). Thus, using the definitions we showed that

$$
\mathcal{O}_{F_{1}^{\prime}} \cap \mathcal{O}_{F_{2}^{\prime}}=\emptyset \text {. }
$$

This establishes that our topology is Hausdorff.

Let now $\bigcup_{i \in I} \mathcal{O}_{F_{i}}=\widehat{E}_{\mathcal{H}}$ be an open covering of $\widehat{E}_{\mathcal{H}}$. We will prove that there exists a finite open subcovering.

If $\widehat{E}_{\mathcal{H}}=\bigcup \mathcal{O}_{F_{i}}$ then there is no $\mathcal{H}$-filter containing all $F_{i}$, which implies that there exist $i_{1}, i_{2}, \ldots, i_{n} \in I$

$$
\begin{aligned}
& D_{i_{1}} \in F_{i_{1}}, D_{i_{2}} \in F_{i_{2}}, \quad D_{i_{n}} \in F_{i_{n}} \text { s.t. } \\
& D_{i_{1}} \cap D_{i_{2}} \cap \cdots \cap D_{i_{n}}=\emptyset
\end{aligned}
$$

(if it would not, the $\mathcal{H}$-filter generated by all $F_{i}$ would exist and satisfy the non empty intersection property.) The above property implies by definition that $\bigcup_{j=1}^{n} \mathcal{O}_{F_{i_{j}}}=$ $\widehat{E}_{\mathcal{H}}$. 


\section{B Comparison of compactifications}

An interesting question is the following: if $\mathcal{F}$ and $\mathcal{G}$ are two compactifying families such that $\mathcal{G} \subset \mathcal{F}$, under which conditions do $\mathcal{G}$ and $\mathcal{F}$ give the same compactification? This is investigated in the following theorem.

Theorem B.1 Let $E$ be a normal topological space and let $\mathcal{F}$ and $\mathcal{G}$ be two compactifying families such that $\mathcal{G} \subset \mathcal{F}$. Then $\mathcal{G}$ and $\mathcal{F}$ yield the same compactification if and only if for any couple $\left(F_{1}, F_{2}\right)$ of elements of $\mathcal{F}$ such that $F_{1} \cap F_{2}=\emptyset$, there exists a couple $\left(G_{1}, G_{2}\right)$ of elements of $\mathcal{G}$ such that $F_{1} \subset G_{1}, F_{2} \subset G_{2}$ and $G_{1} \cap G_{2}=\varnothing$.

Proof Let us first prove the necessity: Let $\left(F_{1}, F_{2}\right)$ be a couple of elements of $\mathcal{F}$ such that $F_{1} \cap F_{2}=\emptyset$ and such that there does not exist a couple $\left(G_{1}, G_{2}\right)$ of elements of $\mathcal{G}$ such that: $F_{1} \subset G_{1}, F_{2} \subset G_{2}, G_{1} \cap G_{2}=\emptyset$.

Let $\mathcal{G}_{1}$ be the set of all elements of $\mathcal{G}$ containing $F_{1}$ and let $\mathcal{G}_{2}$ be the set of all elements of $\mathcal{G}$ containing $F_{2}$.

By hypothesis we know that if $A \in \mathcal{G}_{1}$ and $B \in \mathcal{G}_{2}$ then $A \cap B \neq \emptyset$. Let $\mathcal{U}$ be a $\mathcal{G}$-ultrafilter containing all such intersections. Let us first prove that there is no element of $\mathcal{U}$ which is disjoint from $F_{1}$ (respectively $F_{2}$ ).

If there existed $h \in \mathcal{U}$ s.t. $F_{1} \cap h=\emptyset$, then by property 3 of compactifying families there would exist $h_{1} \in \mathcal{G}, h_{1} \supset F_{1}$ such that $h_{1} \cap h=\emptyset$. But by hypothesis since $h_{1} \in \mathcal{G}, \quad h_{1}$ is also in $\mathcal{U}$ and $h_{1} \cap h=\emptyset$, which is impossible (by construction of $\mathcal{U}$ ), thus all elements of $\mathcal{U}$ intersect $F_{1}$ (respectively $F_{2}$ ). Let $\mathcal{K}_{1}$ be an $\mathcal{F}$-ultrafilter containing $F_{1}$ and $\mathcal{U}$ and $\mathcal{K}_{2}$ an $\mathcal{F}$-ultrafilter containing $F_{2}$ and $\mathcal{U}$. Since $F_{1} \cap F_{2}=$ $\emptyset, \quad \mathcal{K}_{1}$ and $\mathcal{K}_{2}$ are distinct ultrafilters.

Let us now prove that the condition is sufficient:

Let $\mathcal{U}$ be an ultrafilter of elements of $\mathcal{G}$ and $\mathcal{K}_{1}$ and $\mathcal{K}_{2}$ be two $\mathcal{F}$ ultrafilters both containing $\mathcal{U}$. We will prove $\mathcal{K}_{1}=\mathcal{K}_{2}$.

Let us suppose this is not the case. Then there would exist $F_{1} \in \mathcal{K}_{1}, \quad F_{2} \in \mathcal{K}_{2}$ such that $F_{1} \cap F_{2}=\emptyset$ and by hypothesis, there would exist $G_{1}$ and $G_{2}$ elements of $\mathcal{G}$ such that $G_{1} \supset F_{1}, G_{2} \supset F_{2}, G_{1} \cap G_{2}=\emptyset$ but $G_{1} \in \mathcal{K}_{1}$. Thus $G_{1} \in \mathcal{K}_{1} \cap \mathcal{G}$ and $G_{2} \in \mathcal{K}_{2} \cap \mathcal{G}$. Both $\mathcal{K}_{1} \cap \mathcal{G}$ and $\mathcal{K}_{2} \cap \mathcal{G}$ are filters containing $\mathcal{U}$, which is an ultrafilter. Thus $\mathcal{K}_{1} \cap \mathcal{G}=\mathcal{U}=\mathcal{K}_{2} \cap \mathcal{G}$ and $G_{1} \cap G_{2}=\emptyset$ which is impossible. Thus $\mathcal{K}_{1}=\mathcal{K}_{2}$.

Conversely, let $\mathcal{K}$ be an $\mathcal{F}$ ultrafilter. Let us consider $\mathcal{K} \cap \mathcal{G} . \mathcal{K} \cap \mathcal{G}$ is clearly a $\mathcal{G}$-filter.

Let $G \in \mathcal{G}$ such that $G \notin \mathcal{K} \cap \mathcal{G}$. This implies that there exists $F \in \mathcal{K}$ s.t. $G \cap F=\emptyset \quad\left(\mathcal{K}\right.$ is a $\mathcal{F}$-ultrafilter). Then if $G_{1}$ and $G_{2}$ are two elements of $\mathcal{G}$ such that $G_{1} \supset G$ and $G_{2} \supset F$ and $G_{1} \cap G_{2}=\emptyset, G_{2} \in \mathcal{K}$. Thus $G \cap G_{2}=\emptyset$ with $G_{2} \in \mathcal{G} \cap \mathcal{K}$. Thus $\mathcal{K} \cap \mathcal{G}$ is a $\mathcal{G}$-ultrafilter. We thus have a one to one correspondence between $\mathcal{G}$-ultrafilters and $\mathcal{F}$-ultrafilters. The continuity of this correspondence comes from the density of $E$ into both compactifications.

\section{References}

1. Antonevich, A., Radyno, N., Radyno, Y.: About the theory of mnemofunctions and its applications Generalized functions - linear and non linear problems (Novi sad 1998). In: Integral transforms and special functions No 1-4, pp. 123-134 (1998) 
2. Aragona, J., Juriaans, S.O., Oliveira, O.R.B., Scarpalezos, D.: Algebraic and geometric theory of the ring of Colombeau generalized functions. Proc. Edinb. Math. Soc. 51(3), 545-564 (2008)

3. Aragona, J., Juriaans, S.O.: Some structural properties of the topological ring of Colombeau's generalized numbers. Commun. Algebra 29(5), 2201-2230 (2001)

4. Biagioni, H.A.: A nonlinear theory of generalized functions. Lecture Notes in Mathematics, vol. 1421. Springer, Berlin (1990)

5. Burtscher, A., Kunzinger, M.: Algebras of generalized constants with smooth parameter dependence. Proc. Edinb. Math. Soc. 55(1), 105-124 (2012)

6. Colombeau, J.F.: Elementary introduction to new generalized functions. North Holland Math. Studies, vol. 113, (1985)

7. Colombeau, J.F.: Multiplication of distributions: a tool in mathematics, numerical engineering and theoretical Physics. Lecture Notes in Mathematics, vol. 1532. Springer, Berlin Heidelberg (1992)

8. Colombeau, J.F., Oberguggenberger, M.: On a hyperbolic system with a compatible quadratic term: generalized solutions, delta waves, and multiplication of distributions. Commun. Part. Diff. Eq. 15(7), 905-938 (1990)

9. Delcroix, A., Scarpalezos, D.: Asymptotic scales, asymptotic algebras. Integral Transf. Special Funct. 6, 157-166 (1998)

10. Delcroix, A., Scarpalezos, D.: Topology on asymptotic algebras of generalized functions and applications. Monatsh. Math. 129, 1-14 (2000)

11. Delcroix, A., Scarpalezos, D. Sharp topologies on $(\mathcal{C}, \mathcal{E}, \mathcal{P})$-algebras. In: Nonlinear theory of generalized functions (Proc. Vienna, 1997), Grosser, M. e.a. (eds.), Chapman \& Hall (1999), pp. 165-173

12. Delcroix, A., Hasler, M., Pilipović, S., Valmorin, V.: Algebras of generalized functions through sequence spaces algebras, functoriality and associations. Int. J. Math. Sci. 1, 13-31 (2002)

13. Delcroix, A., Hasler, M., Pilipović, S., Valmorin, V.: Sequence spaces with exponent weights. Realization of Colombeau type algebras. Dissertationes Math. 447, 1-73 (2007)

14. Garetto, C.: Topological structures in Colombeau Algebras: topological $\widetilde{\mathbb{C}}$-modules and duality theory. Acta. Appl. Math. 88, 81-123 (2005)

15. Garetto, C.: Topological structures in Colombeau algebras: investigation of the duals of $\mathcal{G}_{c}(\Omega), \mathcal{G}(\Omega)$ and $\mathcal{G}_{\mathcal{S}}\left(\mathbb{R}^{n}\right)$. Monatsh. Math. 146, 203-226 (2005)

16. Gillman, L., Jerison, M.: Rings of Continuous Functions. Princeton, N.J. Van Nostrand (1960)

17. Grosser, M., Kunzinger, M., Oberguggenberger, M., Steinbauer, R.: Geometric Theory of Generalized Functions. Kluwer, Dordrecht (2001)

18. Khelif, A., Scarpalezos, D., Vernaeve, H.: Asymptotic Ideals (ideals in the ring of Colombeau generalized constants with continuous parametrization). Commun. Algebra 42(6), 2721-2739 (2014)

19. Kunzinger, M., Oberguggenberger, M.: Characterization of Colombeau generalized functions by their point values. Math. Nachr. 203, 147-157 (1999)

20. Bang-He, L.: Li Ya-Qing. New generalized functions in non standard framework, Acta mathematica sinica (english translation) (1992)

21. Nedeljkov, M., Pilipović, S., Scarpalezos, D.: The linear theory of Colombeau generalized functions, Pitman Res. Not. Math. 385, Longman, Harlow (1998)

22. Oberguggenberger, M.: Multiplication of distributions and applications to partial differential equations, Pitman Res. Not. Math. 259. Longman, Harlow (1992)

23. Oberguggenberger, M., Todorov, T.: An embedding of Schwartz distributions in the algebra of asymptotic functions. Int. J. Math. Sci. 21(3), 417-428 (1998)

24. Oberguggenberger, M., Wang, Y.G.: Generalized Solutions to Conservation Laws. Zeitschr. Anal. Anw. 13, 7-18 (1994)

25. Scarpalezos, D.: Colombeau generalized functions: Topological structures; microlocal properties. A simplified point of view. Prepublications mathématiques de l'U.R.A. "théories géometriques" (Université Paris 7), (1993)

26. Scarpalezos, D.: Some remarks on functoriality of Colombeau's construction: topological and microlocal aspects and applications. Int. Transf. Spec. Fct. 6, 295-307 (1998)

27. Scarpalezos, D.: $m$-reduced asymptotic constructions, fields and generalized functions. In: Nonlinear algebraic analysis and applications (Proc. Guadeloupe, 2000), Delcroix, A. e.a. (eds.), Cambridge Scientific Publishers (2004)

28. Vernaeve, H.: Ideals in the ring of Colombeau generalized numbers. Commun. Algebra 38(6), 2199$2228(2010)$ 
Publisher's Note Springer Nature remains neutral with regard to jurisdictional claims in published maps and institutional affiliations. 